\title{
Strong Materials
}


An Experimental Study on Adhesive or Anti-adhesive, Bio-inspired Experimental Nanomaterials 
Chapter 8

\title{
Evidence of the Most Stretchable Egg Sac Silk Stalk of the European Spider of the Year Meta Menardi
}

\begin{abstract}
Spider silks display strong mechanical properties, even with observed differences between species and within the same species. While many different types of silks have been tested, the mechanical properties of silk stalks taken from egg sacs of the cave spider Meta menardi have not yet been analyzed. Meta menardi has recently been chosen as the "European spider of the year 2012" from the European Society of Arachnology. Here we report a study in which silk stalks were collected directly from several caves in north-west Italy. Field emission scanning electron microscope (FESEM) images show that stalks consist of a large number of threads, each with diameter of $6.03 \pm 0.58 \mu \mathrm{m}$. The stalks were strained at a constant rate of $2 \mathrm{~mm} / \mathrm{min}$ using a tensile testing machine. The observed maximum stress, strain, and toughness modulus, defined as the area under the stress-strain curve, are $0.64 \mathrm{GPa}, 751 \%$, and $130.7 \mathrm{MJ} / \mathrm{m}^{3}$, respectively. To our knowledge, such a huge observed elongation has never been reported for egg sac silk stalks and suggests a significant unrolling microscopic mechanism of the macroscopic stalk that, as a continuation of the protective egg sac, is expected to be composed of very densely and randomly packed fibers.

Weibull statistics were used to analyze the results from mechanical testing, and an average value for the Weibull modulus $(m)$ is found to be in the range of $1.5-1.8$ with a Weibull scale parameter $\left(\sigma_{0}\right)$ in the range of $0.33-0.41 \mathrm{GPa}$, showing a high coefficient of correlation $\left(R^{2}=0.97\right)$.
\end{abstract}

\subsection{Introduction}

Spider silks generally display strong mechanical properties (Brunetta, 2010) and have been studied extensively during the last five decades. In particular, dragline silk is noted for its unique strength and toughness. Because of the complex structure of spider silk, large scale synthetic production remains a challenge and can only be achieved through controlled self-assembly of macromolecular components with nanoscale precision (Keten, 2010a).

(cc) BY-NC-ND 
Individual spiders spin 'toolkits' of seven to eight different types of silks, each of which is generated from its own discrete gland(s) and spigot(s) (Blackledge, 2006). Each type of spider silk has a unique chemical composition, molecular structure, and material properties (Blackledge, 2011). Orbwebs, for example, are composite structures built from multiple types of silks, each with its own unique molecular structure and mechanical function (Blackledge, 2011).

The most studied silk type is dragline silk, which is produced in the major ampullate gland. As the name suggests, dragline silk is used as a lifeline by most spiders moving through their environment and forms the backbone of most webs (Blackledge, 2011). Minor ampullate glands produce threads that are sometimes added to major ampullate draglines or temporary spirals of the orbweb acting as a scaffold for web construction. Aciniform glands produce silk used for wrapping prey and egg case construction, and its fibers are more stretchable and tougher than dragline silk (Rousseau, 2009). Flagelliform glands are unique to araneoidorbweaving spiders and are used in the production of catching spiral silk. In some derived taxa (like cobweb spinning theridiids) this type of silk is used to wrap prey (Eberhard, 2010). Aggregate glands produce the glue coating on viscid capture threads and are unique to araneoid spiders, whereas piriform glands are used to cement threads to substrata as well as to form silk junctions by forming attachment disks (Blackledge, 2011).

It is widely accepted that the tubuliform (or cylindrical) glands play a major role in silk production for egg sacs (Kovoor, 1987; Foelix, 1996; Foradori, 2002; Craig, 2003), and it is likely that some spiders produce egg sac silk exclusively in these glands. Tubuliform silk is produced solely by adult orbweaving females. Egg sacs are complex, layered structures containing fibres from several different glands (Gheysens, 2005; Hajer, 2009; Vasanthavada, 2007). This complexity creates confusion about how tubuliform silk is utilized. However, the morphology of the silk is quite distinctive because the glands produce large fibers with an irregular surface that is unlike any other silk. Moreover, the left and right fibers are coated with a gluey secretion that causes them to adhere together (Gheysens, 2005). The mechanical behaviour of the silk is distinct in displaying a prominent yield followed by a long low modulus extension (Keten, 2010b; Blackledge, 2011; Van Nimmen, 2006).

In orbweb spiders, the spinnerets are three paired appendage-like organs on the abdomen, each of which contains dozens to hundreds of spigots connected to their own internal silk-producing glands (Fig.8.1) (Vehoff, 2007). A single spider is therefore capable of producing multiple silk threads of many kinds, and the arrangement of spigots on the spinnerets appears to relate functionally to how different silks are used together (Eberhard, 2010). Dragline silk, flagelliform silk, aggregate silk, and aciniform silk have been extensively characterized in Argiope trifasciata (Forsskål) (Platnick, 2011; Perez-Rigueiro, 2001; Elices, 2005; Poza, 2002; Guinea, 2003; Hayashi, 2004); Araneus diadematus (Linnaeus) (Van 
Nimmen, 2005a; Madsen, 1999; Van Nimmen, 2005b; Vollrath, 2001; Gosline, 1999; Köhler, 1995; Römer, 2008; Gosline, 1986; Ortlepp 2008; Shao, 2008); Argiope argentata (Fabricius) (Blackledge, 2006; Swanson, 2006); Argiope bruennichi (Scopoli) (Zhao, 2006); Araneus gemmoides Chamberlin \& Ivie (Swanson, 2006; Stauffer, 1994); Larinioides (=Araneus) sericatus Clerck (Denny, 1975); Nephila edulis (Labillardière) (Madsen, 1999; Vollrath, 2001); Nephila clavipes (Linnaeus) (Swanson, 2006; Stauffer, 1994; Dunaway, 1995; Cunniff, 1994; Vollrath, 1996); Nephila pilipes Fabricius (Dunaway, 1995); Nephila madagascariensis (= N. inaurata madagascariensis) (Vinson) (Gosline, 1986); Lactrodectus hesperus Chamberlin \& Ivie (Swanson, 2006; Moore, 1999); Leucauge venusta Walckenaer (Swanson, 2006); Plectreurys tristis Simon (Swanson, 2006); Kukulcania hibernalis Hentz (Swanson, 2006); Salticus scenicus (Clerck) (Ortlepp, 2008). These studies have shown that various silk types, produced by different glands, have very different mechanical properties (Van Nimmen, 2005a; Van Nimmen, 2005b; Stauffer, 1994), giving the threads different characteristics depending on their respective function (Foelix, 1996) that may vary according to different species. Variability in the mechanical properties of spider silk is very important. Spider silk is central to many aspects of spider biology and ecology, from communication to prey capture. Spiders are the only animals which use silk in almost every part of their lives. Because of its importance, it has presumably been subjected to strong selective pressures during the 400 million years of spider evolution and can be regarded as one of the keys to spides evolutionary success (Craig, 2003; Sensenig, 2010).

It has been demonstrated that silk properties in terms of different reeling methods (Swanson, 2006; Boutry, 2011), environmental conditions (Foelix, 1996; Guinea, 2003), and types of silk (e.g. dragline, viscid or egg sac silk) (Van Nimmen, 2005a; Van Nimmen, 2005b; Stauffer, 1994) are species-specific and lead to silk-based peptide fibrils or protein aggregates with different structural and mechanical properties. For example, different reeling speeds cause a variation in the diameter of dragline thread (Vollrath, 2001) and thus the stress-strain curve varies depending on the thickness of the thread. Spider dragline silk was tested in a wet environment to show that moisture induces supercontraction for higher than $70-75 \%$ humidity. When a thread is exposed to moisture, stresses quickly build up and tighten the thread (Guinea, 2003). By varying the conditions under which the spiders were kept (different reeling speeds, starvation periods), it was observed that dragline silk has different mechanical properties and varies on an interspecific, intraspecific, and intra-individual level (Madsen, 1999).

All silks are proteinaceous and belong to the general class of hierarchical protein materials. Each thread of spider silk is a composite of semi-amorphous $\alpha$-chains and $\beta$-pleated nanocrystals (Keten, 2010b). In the orb web spider Araneus diadematus (the common European garden spider), the $\beta$-sheets consist of a series of highly conserved poly-Ala repeats and are stacked to form protein 
crystals; these crystals are embedded in a matrix of loosely arranged glycine-rich amino acids (Gosline, 1999). The protein crystals are held together by hydrogen bonds, one of the weakest chemical bonds, which serve an important role in defining the mechanical properties of silk. When an external force is applied, loose amino acids stretch and are straightened from a disordered position, whereas the $\beta$-sheets are subject to tensile forces (Krasnov, 2008). The $\beta$-sheet rich crystalline units are responsible for the toughness of the silk thread, and the remaining apparently amorphous regions have a rubber-like behavior (Gosline, 1984). One study uses a simple coarse-grained model to simulate the mechanical deformation of silk in which the silk constitutive unit was a combination of two domains representing $\alpha$-chains and $\beta$-pleated sheets (Nova, 2010). The stressstrain curve of the simulation has a similar shape to that of silk.

The studies on dragline silk have provided an opportunity to find a natural fiber with strong tensile properties in terms of large deformation (Blackledge, 2006; Foelix, 1996; Perez-Rigueiro, 2001; Elices, 2005; Poza, 2002; Hayashi, 2004; Van Nimmen, 2005a; Madsen, 1999; Van Nimmen, 2005b; Vollrath, 2001; Gosline, 1999; Köhler, 1995; Römer, 2008; Gosline, 1986; Ortlepp, 2008; Swanson, 2006; Zhao, 2006; Stauffer, 1994; Denny, 1976; Dunaway, 1995; Cunniff, 1994; Moore, 1999; Agnarsson, 2010). A recent study has discovered a dragline silk that is twice as tough as any other previously described silk. This silk belongs to Caerostris darwini Kuntner \& Agnarsson, a spider which constructs its orb web suspended above streams, rivers, and lakes (Agnarsson, 2010). To thoroughly understand all the various properties of spider silk, we must characterize the different kinds of silk.

The stress-strain behavior of the egg sac silk of Araneus diadematus (Van Nimmen, 2005b) presents a logarithmic behavior, which is completely different from the behavior of dragline and viscid silk. The same can be said about the egg sac silk of Argiope bruennichi (Zhao, 2006). The stress-strain curves of the egg sac silk start with a small elastic region and then present an extremely flat plastichardening region (Van Nimmen, 2005b). The breaking strain is roughly the same as that of the dragline, but tensile strength is about 3 to 4 times lower. Egg case silk has an initial modulus, a measurement of the stiffness of the fiber, which is significantly higher than that of dragline thread. These differences are partly due to the different amino acid compositions in the silks. To our knowledge, few studies have been conducted on stalks of egg sac silk. In general, each egg sac consists of two major parts that can be distinguished by the naked eye, an egg sac case and a stalk. The egg sac case houses eggs, while the stalk attaches the cocoon to the substrate (Hajer, 2009). In the literature, the strain of spider egg sac silk is in the range from $19 \%$ for Araneus gemmoides (Stauffer, 1994) to $29 \%$ for Argiope argentata (Blackledge, 2006), showing an average value of $26 \%$; the average stress is $1.1 \mathrm{GPa}$ with a minimum value of $0.3 \mathrm{GPa}$ for Araneus diadematus (Van Nimmen, 2005b) and the maximum stress of $2.3 \mathrm{GPa}$ for Araneus gemmoides (Stauffer, 1994). 
One study pulled bundles of 100 dragline and minor ampullate silk threads at constant speeds (Stauffer, 1994). They observed that physical interactions between fibers influence elongation and thus increase the stretching capabilities of the bundle compared to that of a single fiber. They observe that Nephila clavipes dragline silk shows almost double the final stress value compared to the same silk of Araneus gemmoides, whereas the minor ampullate silk shows roughly the same final stress value (Stauffer, 1994).

The cave spider Meta menardi (Latreille) is generally found in dark and humid places like caves and mines throughout the northern hemisphere, from northern Europe to Korea and northern Africa (Platnick, 2011; Lepore, 2012b). Meta menardi has recently been chosen as the "European spider of the year 2012" from the European Society of Arachnology. Since no engineering studies of the egg sac of the cave spider Meta menardi yet exist and just a few studies have been focus on egg sacs, we conducted tensile tests on stalks of egg sac silk. We tested the stalk which connect the egg sacs of Meta menardi to the ceiling of caves (the arrow, in Fig.8.2, indicates such sample). In total 15, stalks were found and were pulled until they broke. Samples were viewed under FESEM to analyze the fracture surfaces and measure the diameter of the stalk. To visualize the thread stacking in each stalk, a Focused Ion Beam (FIB) was used to cut the stalk. Using FESEM micrographies of cross-sections of the FIB-cut stalk and the processing software Image] 1.410, the real diameter and the exact number of single threads in each stalk were measured, improving previous accuracy (Poza, 2002). Thus, the stress-strain curves and the Weibull shape and scale parameters of the egg sac silk stalk of Meta menardi are determined here.

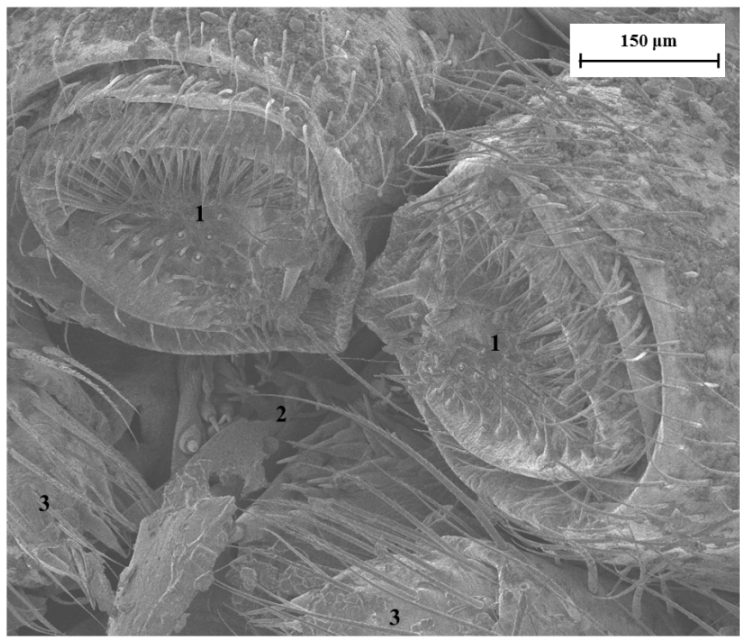

Figure 8.1 FESEM image of the spinnerets of Meta menardi (1. Anterior lateral; 2. Posterior median; 3. Posterior lateral). 


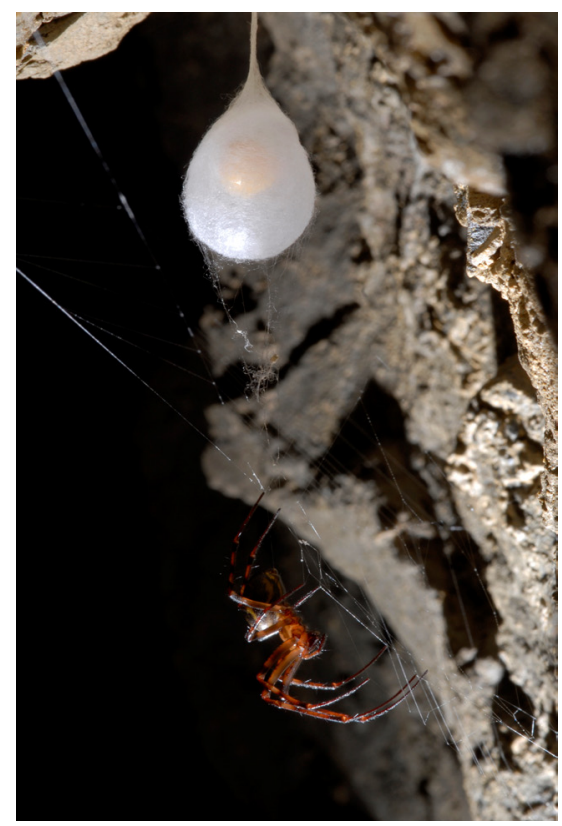

Figure 8.2 Egg sac of the spider Meta menardi. Photo by Francesco Tomasinelli (2009).

\subsection{Materials and Methods}

Note that: no specific permits were required for the described field studies; the location is not privately-owned; the field studies did not involve endangered or protected species.

\subsubsection{Tensile Testing}

We identified caves in Piedmont (a north-western region of Italy) to search for Meta menardi egg sacs. The egg sacs are generally spun at the end of summer and hatch in late winter. Fifteen stalks of the egg sacs were taken from the caves in which they were found (Table 8.1). Since the egg sacs were collected in their natural habitat, the measured mechanical stress-strain behavior of the silk would most likely represent real silk characteristics more accurately than that produced by lab-reared spiders.

We collected fifteen stalks of the egg sacs in three different caves: four in Grotta Inferiore del Pugnetto, three in Grotta del Bandito, and eight in Grotta di 
Chiabrano. The spiders of this species are generally found in dark areas close to cave openings, where temperature and humidity are still influenced by external conditions. The egg sacs hung from the ceilings of the caves with a bundle of threads (stalk) and were generally found in ventilated areas. The surveys were done on three separate days. The stalks of the egg sacs were carefully took from the cave ceilings and glued only by the ends to $30 \mathrm{~mm} \times 50 \mathrm{~mm}$ cardboard holders. Each holder had a $20 \mathrm{~mm} \times 20 \mathrm{~mm}$ hole in its center so that the stalks could be suspended, transported while maintaining original tension, and mounted on the testing machine without being damaged. All tests were done in the Laboratory of Bio-inspired Nanomechanics "Giuseppe Maria Pugno" (Politecnico di Torino, Italy) with an air temperature of $22 \pm 1^{\circ} \mathrm{C}$ and $31 \pm 2 \%$ relative humidity.

Tensile tests were conducted on thirteen of the fifteen specimens, whereas the remaining two specimens were considered representative of the tested samples and examined under the FESEM and FIB. The tensile tests were conducted using a testing machine (Insight $1 \mathrm{kN}, \mathrm{MTS}$, Minnesota, USA), equipped with a $10 \mathrm{~N}$ cell load with pneumatic clamps (closure pressure of $275.6 \mathrm{kPa}$ ). The cardboard holders were placed between the clamps with double-sided tape defining an initial length $l_{0}$ in the range from 18 to $19 \mathrm{~mm}$. Once the holders were in place, the clamps were brought to zero tension and then the sides of the holders were cut, leaving the stalk loose between the clamps. The specimens were pulled until they completely broke at a constant rate of $2 \mathrm{~mm} / \mathrm{min}$, consistent with the parameter setting of previous studies (Giunea, 2003; Madsen, 1999; Van Nimmen, 2005b; Vollrath, 2001; Gosline, 1986; Zhao, 2006; Stauffer, 1994; Álvarez-Padilla, 2009).

Table 8.1 List of the caves visited for the collection of the samples with collection date and number of samples.

\begin{tabular}{|l|l|l|l|l|l|}
\hline Cave name & $\begin{array}{l}\text { Speleological } \\
\text { cadastre number }\end{array}$ & Municipality & Province & Date & $\begin{array}{l}\text { Number of } \\
\text { samples }\end{array}$ \\
\hline $\begin{array}{l}\text { Grotta del } \\
\text { Bandito }\end{array}$ & $1002 \mathrm{Pi} / \mathrm{CN}$ & Roaschia & Cuneo & $02 / 2011$ & 3 \\
\hline $\begin{array}{l}\text { Grotta } \\
\text { inferiore del } \\
\text { Pugnetto or } \\
\text { Tana del lupo }\end{array}$ & $1502 \mathrm{Pi} / \mathrm{TO}$ & Mezzenile & Torino & $02 / 2011$ & 4 \\
\hline $\begin{array}{l}\text { Grotta di } \\
\text { Chiabrano or } \\
\text { Tuna del Diau }\end{array}$ & $1621 \mathrm{Pi} / \mathrm{TO}$ & Perrero & Torino & $02 / 2011$ & 8 \\
\hline
\end{tabular}

The computer program TestWorks 4 (MTS, Minnesota, USA) recorded the applied tensile force data, and the stress-strain curves were computed using the estimation of the real diameter and the exact number $n$ of single threads at the cross-section of each stalk. Stress $\sigma$, strain $\varepsilon$, and modulus $E$ were calculated using the following equations $(1,2,3)$ : 


$$
\begin{aligned}
& \sigma=\frac{F}{\sum_{\mathrm{i}=1}^{\mathrm{n}} A_{\mathrm{i}}}=\frac{F}{A_{\mathrm{b}}} \\
& \varepsilon=\frac{\Delta l}{l_{0}} \\
& E=\left.\frac{d \sigma}{d \varepsilon}\right|_{0}
\end{aligned}
$$

where $F$ is the force measured by the testing machine, $A_{b}$ is the initial crosssectional area of the stalk (given as the initial cross-sectional area $A_{i}$ of a single thread multiplied by the number $n$ of stalk threads), $l_{0}$ is the initial length of the stalk, and $\Delta l$ is the change in stalk length during the test. The area under the stress-strain curve gives the energy required to break the material, and this variable can be used to quantify toughness. Spider silk dissipates energy in volume, and so the classical fracture toughness cannot be defined, which suggest significant intrinsic toughening mechanisms.

Weibull statistics were applied to the stress results of the tensile tests. The probability of failure $P$ for a stalk is defined as:

$$
P(\sigma)=1-e^{-\left(\frac{\sigma}{\sigma_{0}}\right)^{m}}
$$

where $\sigma$ is the applied stress, $m$ is the Weibull shape parameter, or Weibull modulus, and $\sigma_{0}$ is the Weibull scale parameter. The cumulative probability $P_{i}\left(\sigma_{1}\right)$ can be obtained experimentally as:

$$
P_{\mathrm{i}}\left(\sigma_{\mathrm{i}}\right)=\frac{\mathrm{i}-1 / 2}{N}
$$

where $N$ is the total number of measured fracture stresses $\sigma_{1}$ ranked in ascending order.

\subsubsection{FESEM and FIB Characterization}

Each stalk was cut by FIB (FEI Quanta 3D FEG, at $5 \mathrm{kV}$ ). The real diameter and the exact number of single threads in each stalk was determined using FESEM (FEIInspect ${ }^{\mathrm{TM}} \mathrm{F} 50$, at $1-2 \mathrm{kV}$ ) micrographies of the FlB-cut stalk cross-section and the processing software Image] 1.410 . 


\subsection{Results}

We performed tensile tests on the egg sac silk stalks of Meta menardi. The 13 stalks that we were tested were divided into two groups depending on the stalk type. Two types of stalk could be macroscopically distinguished: "cable" type (group A) and "ropey" type (group B). The "cable" type stalk consists of a series of threads tightly packed together to form a very compact structure (Fig.8.3 a), whereas the threads are not compact in the "ropey" type stalk (Fig.8.3 b). There were 4 stalks in group A and 6 stalks in group B. The remaining stalks were discarded as they did not reveal concrete information in terms of tensile strength. The performed tensile tests show significantly different values for stress, strain, and modulus; Weibull statistics were applied to interpret the results.

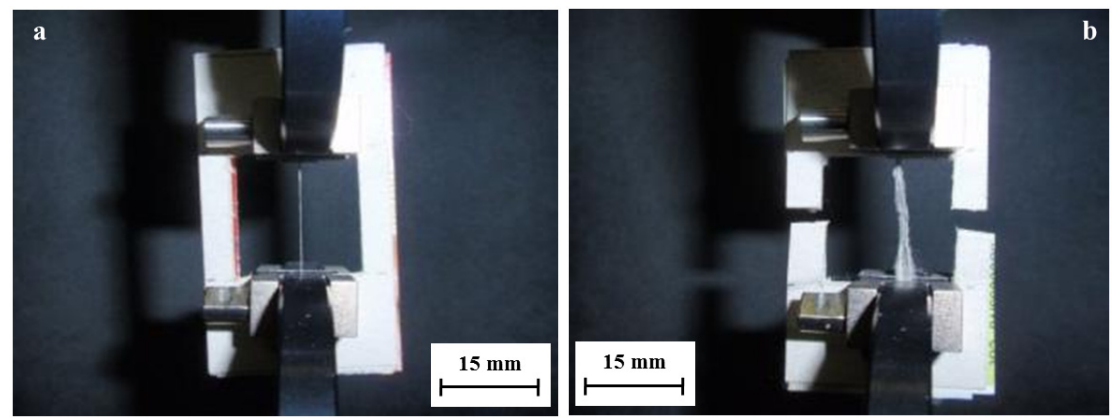

Figure 8.3 Distinction of the stalk types: cable-like (Group A) (a) and ropey (Group B) (b).

The FESEM images show that the threads composing the stalks are all of similar diameter and are parallel-oriented (Figs.8.4 a, b, c). Stalk ends were clamped between the pneumatic clamps with double-sided tape at a closure pressure of $275.6 \mathrm{kPa}$, which is a high pressure compared with the testing forces. Consequently, the macroscopic unraveling or slipping of the stalk or the cardboard holders between the clamps were impossible, so only sufficient bundle stretching is allowed. Moreover, no additional length is available for sliding after the clamps so we can exclude artifacts in our observations.

The diameter of a single thread is $6.03 \mu \mathrm{m}$ (Fig.8.4 d), which is close to the upper value of the range from 1 to $6 \mu \mathrm{m}$ previously indicated (Foelix, 1996), whereas the diameter of the bundles fall in the range from 200 to $300 \mu \mathrm{m}$. The FIB images allow us to observe how many silk threads compose each stalk and thus calculate the real thread cross-sectional area. Using the FESEM, we see that each stalk is composed of an average of 150 single silk threads, corresponding to an effective cross section of $4283.67 \mu \mathrm{m}^{2}$. 


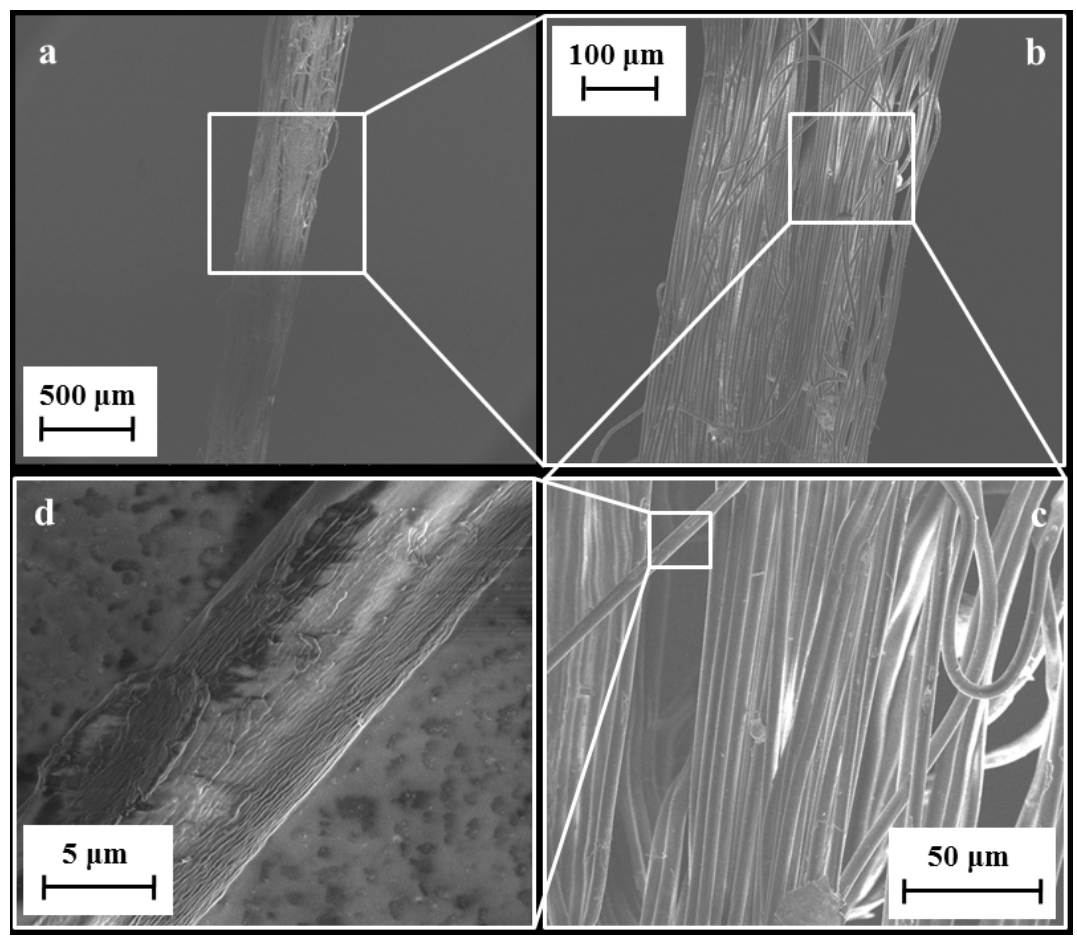

Figure 8.4 FESEM characterization of the silk stalk at different magnifications.

From the various tensile tests, and although the data is scattered, we calculate the average failure stress of $0.355 \mathrm{GPa}$ for group $\mathrm{A}$ and $0.286 \mathrm{GPa}$ for group $\mathrm{B}$. The average failure strain is calculated to be $318 \%$ for group A and $227 \%$ for group B. The average values of toughness are $76.5 \mathrm{MJ} / \mathrm{m}^{3}$ for group $A$ and $51.3 \mathrm{MJ} / \mathrm{m}^{3}$ for group $B$. The Young's modulus is calculated as the initial slope of the stress-strain curve, which is equal to 20.4 GPa for group A and 22.46 GPa for group B. Fig.8.5 a, b shows the various characterized stress-strain curves.

The stress-strain curves show different shapes, which is caused by the varying number of threads that compose each stalk. The curves show a small initial elastic region which reaches a maximum stress and then drops quickly to very low values. It continues to large strains until failure is reached, in some cases through a series of peaks caused by the breaking of single or a small number of threads in the stalk. The strain values differed but were all above $20 \%$, with some stalks reaching $300 \%$ strain or more before breaking. Two tests were pulled to an extraordinary length; the maximum strain to which they were subjected was $751 \%$ for stalk $A 2$, corresponding to a toughness value of $130.7 \mathrm{MJ} / \mathrm{m}^{3}$ (solid line in Fig.8.5 a), and $721 \%$ for stalk B4, corresponding to a toughness value of $117.4 \mathrm{MJ} / \mathrm{m}^{3}$ (solid line in Fig.8.5 b). 
$\mathbf{a}$
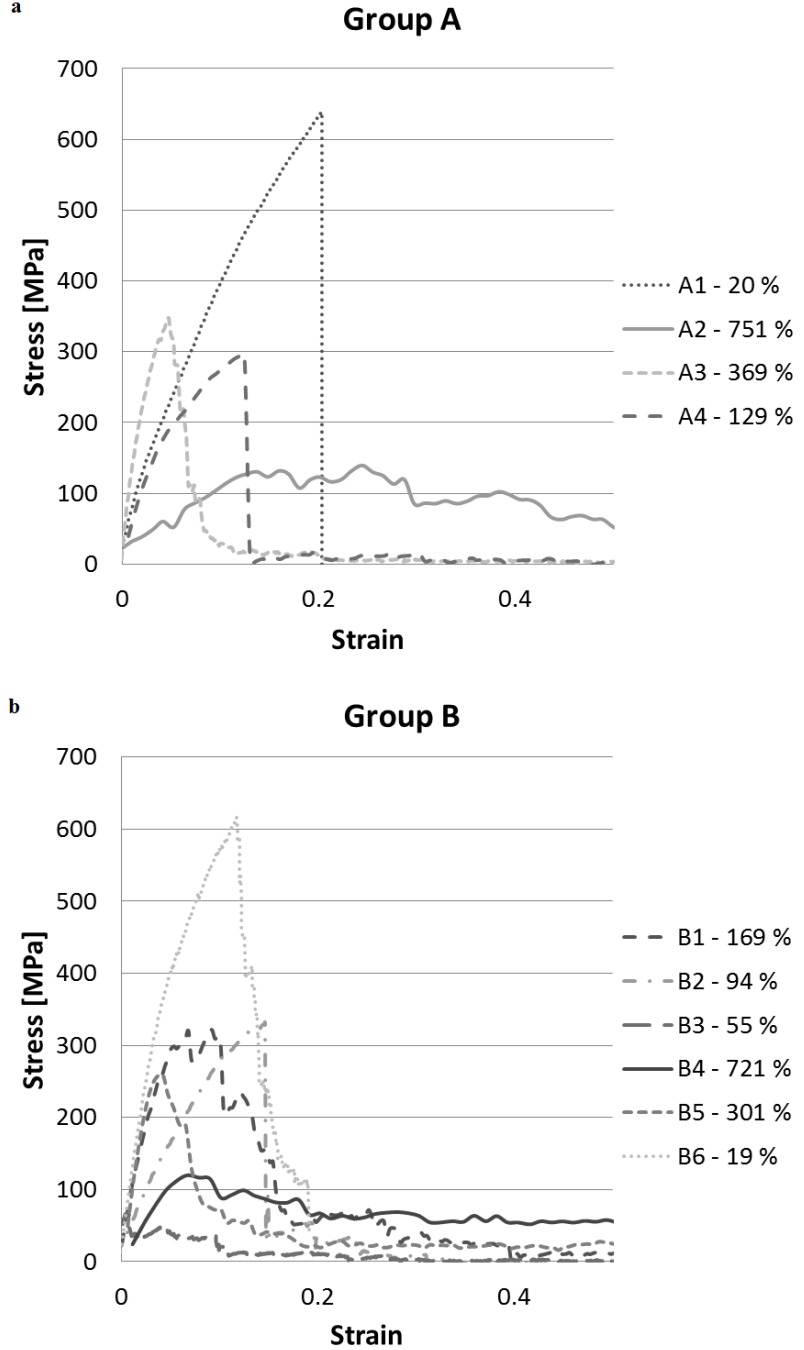

Figure 8.5 Stress-strain curves for group A (a) or B (b) stalks.

Following Weibull statistics, we apply Eq. (4) to the set of fracture stresses of the egg sac silk stalks of Meta menardi reported in Table 8.2. The Weibull modulus $\mathrm{m}$, an index of the dispersion of the stress distribution, is 1.8 for group A (Fig.8.6 a) and 1.5 for group B (Fig.8.6 b), whereas $\sigma_{0^{\prime}}$ an index of the mean value of the stress distribution, is equal to $0.409 \mathrm{GPa}$ for group $A$ and $0.326 \mathrm{GPa}$ for group B. Note that the correlation coefficient is high $\left(R^{2}=0.97\right)$ for both the groups. 


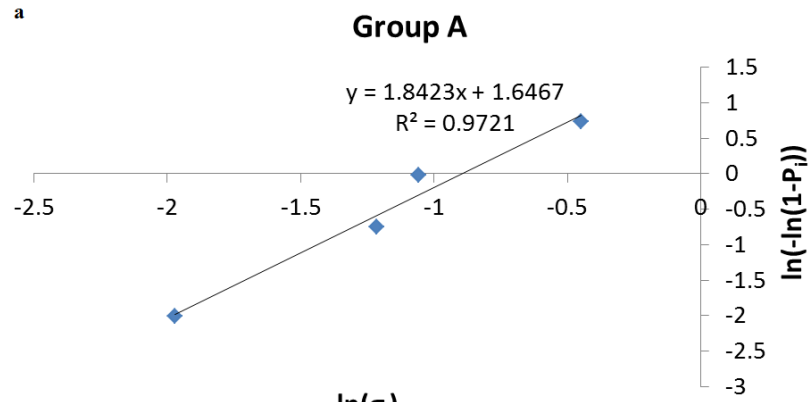

$\ln \left(\sigma_{\mathrm{i}}\right)$

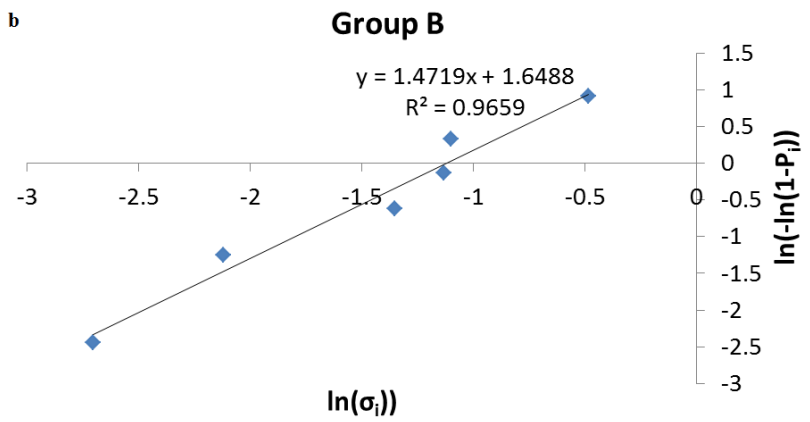

Figure 8.6 Weibull statistics for stress of group A (a) and B (b) stalks.

Table 8.2 The measured stress of each stalk in ascending order.

\begin{tabular}{|c|c|c|c|}
\hline \multicolumn{2}{|c|}{ Group $A$} & \multicolumn{2}{c|}{ Group B } \\
\hline Test ${ }^{\circ}$ & Stress (GPa) & Test $n^{\circ}$ & Stress (GPa) \\
\hline $1(\mathrm{~A} 2)$ & 0.139 & $1(\mathrm{~B} 3)$ & 0.067 \\
\hline $2(\mathrm{A3})$ & 0.297 & $2(\mathrm{~B} 4)$ & 0.120 \\
\hline $3(\mathrm{~A} 4)$ & 0.347 & $3(\mathrm{~B} 5)$ & 0.259 \\
\hline $4(\mathrm{~A} 1)$ & 0.639 & $4(\mathrm{~B} 1)$ & 0.322 \\
\hline & & $5(B 2)$ & 0.332 \\
\hline & & $6(B 6)$ & 0.617 \\
\hline
\end{tabular}

\subsection{Discussion}

Previously, scientists have focused on different types of silk and mechanically characterized them. Only a few studies on tensile tests conducted on egg sac silk have been performed, particularly on Argiope argentata (Blackledge, 2006), Araneus diadematus (Van Nimmen, 2005b), Nephila madagascariensis (Gosline, 
1986), Argiope bruennichi (Zhao, 2006), Araneus gemmoides, and Nephila clavipes (Stauffer, 1994). In addition, the genuses Nephila, Araneus, and Meta belong to three related families of orb web weavers (Nephilidae, Araneidae, and Tetragnathidae, respectively (Platnick, 2011)) and thus general trends can be seen (Xiao, 2008). The shapes of the observed stress-strain curves have a similar shape to that of carbon nanotube (CNT) bundles (Bosia, 2010; PerezRigueiro, 2000). These curves present a series of kinks or load drops, which are an indication of sub-bundle failures when a bundle is pulled parallel to its axis. Our data also have a series of kinks, indicating that the failure of the bundle at its peak load occurs with the fracture of sub-bundles. Although the curves are similar to those of CNT bundles, they are completely different from those of dragline silk bundles and egg sac silk stalks (Stauffer, 1994). Comparing their results to ours, we see that their failure stresses and toughness are much higher.

The $\beta$-sheet nanocrystals are held together by hydrogen bonds, one of the weakest chemical bonds. When a thread is pulled, the force peaks in the force-displacement graph confirm that the hydrogen bonds break and reform at an adjacent hydrogen bond ring. This occurs by preserving the initial sidechain orientation and shifting or by rotating and forming an opposite sidechain orientation. This bond breaking leads to a series of force peaks in the mechanical response and increases the total dissipated energy (Keten, 2010b). The size of the $\beta$-sheet nanocrystals influences the tensile response of a silk thread; consequently, the smaller the crystals, the greater the strength and toughness of the thread. As mentioned above, the fibers are made up of semiamorphous $\alpha$-chains and $\beta$-pleated sheets which are embedded in a rubber-like matrix. Images from the FESEM further show that the fibers consist of 2 layers (Vollrath, 1996): an inner layer and an outer coating. It seems that some fibers have a polymeric-like fracture surface and some have a more regular surface. This second case is probably due to the different crystals composing the fiber. In fact, $\beta$-sheets are crystal-like and are responsible for the toughness of the thread. They also have a more fragile rupture. On the other hand, we can assume that some fibers have a very ductile break caused by amorphous rubber-like regions (Fig.8.7 a, b).

Having cut our stalk with FIB, we are able to observe the cross section of the stalks at a SEM eye angle of $52^{\circ}$ (Fig.8.8 a, b, c) and from the top (Fig.8.8 d). Each stalk is composed of a series of single silk threads which, when pulled, stack together to form what we initially hypothesized as a cylindrical cable. The diameters of the egg sac silk threads $(\sim 6 \mu \mathrm{m})$ are slightly smaller than those of egg sac silk of Nephila clavipes ( 7 $\mu \mathrm{m})$ (Stauffer, 1994) but equal to those of Argiope bruennichi (Zhao, 2006) and still much larger than the dragline silk $(\sim 1.4 \mu \mathrm{m})$ of the same species. For comparison, the diameters of dragline silk and minor ampullate in Nephila clavipes and Araneus gemmoides are estimated to be 3 and $2.5 \mu \mathrm{m}$ (Stauffer, 1994), and 2.5 and $2 \mu \mathrm{m}$ (Zhao, 2006), respectively. 
An Experimental Study on Adhesive or Anti-adhesive, Bio-inspired Experimental Nanomaterials

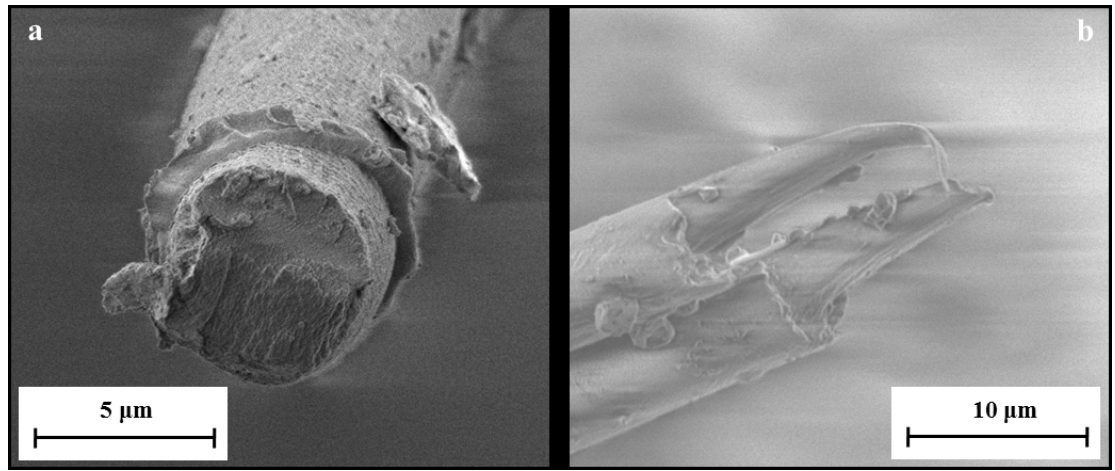

Figure 8.7 Detailed views of fracture surfaces of broken silk fibers.

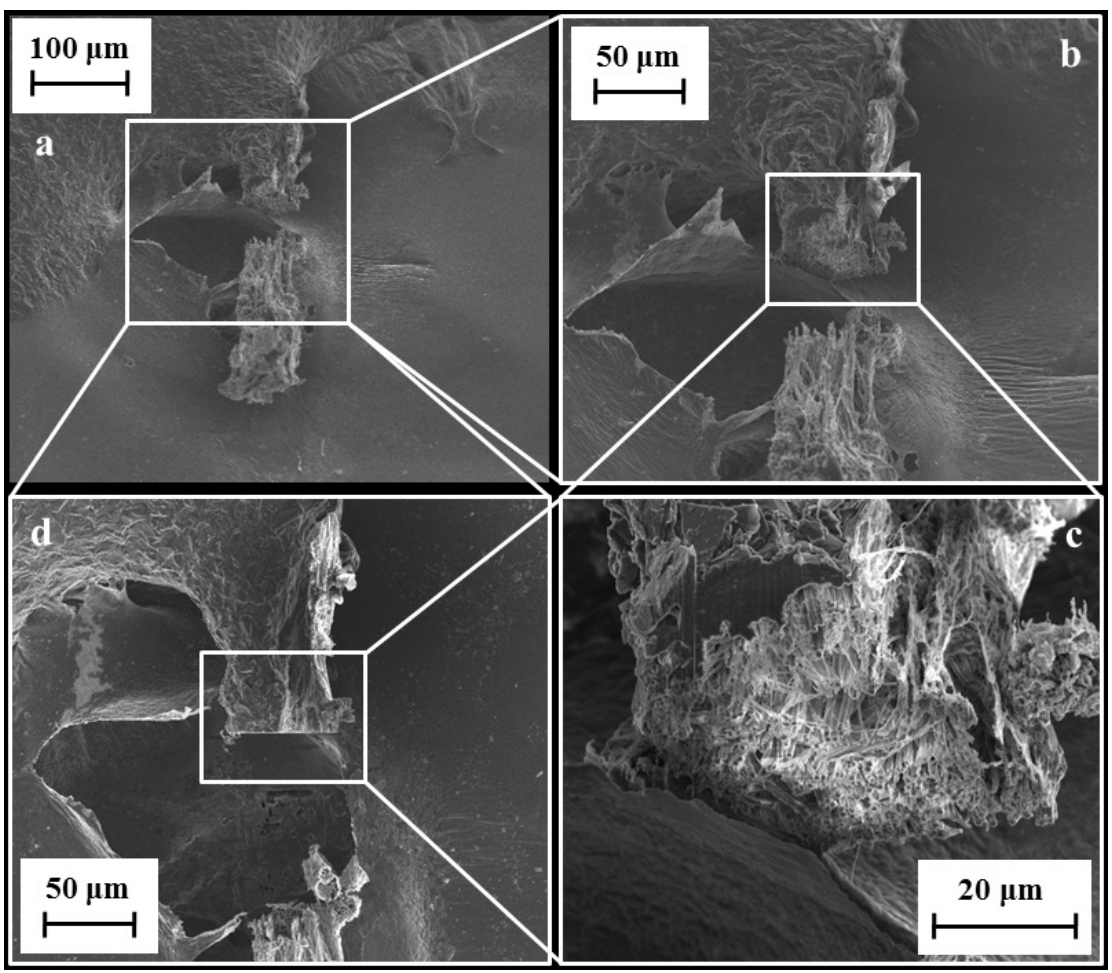

Figure 8.8 FESEM characterization of the stalk cut with FIB: $(a, b, c)$ at an eye angle of $52^{\circ}$ and (d) from the top. 
The strains sustained by our fibers are impressively high; some stalks were pulled to more than $200 \%$ their original length, reaching values of $721-751 \%$, which have not yet been observed in any spider single thread or stalk of egg sac silk. Such enormous elongations suggest a huge unrolling mechanism in the stalk.

In Figs.8.9-8.11, we report toughness, ultimate stress, and ultimate strain, respectively, for different types of spider silks; specifically, in Fig.8.11 our record of ultimate strain emerges. The reason for this very high strain is unknown but might be caused by an interaction and different disposition of the $\alpha$-chains and $\beta$-pleated sheets within the fibers, thus allowing them to stretch to such high strain values. As stated in the introduction, it has been observed that physical interactions between fibers can influence the elongation data and increase the stretching capabilities of the stalk compared to those of a single fiber (Stauffer, 1994). We see that extreme strain of the stalks can be caused by macroscopic unraveling of the stalk itself. The failure strains of the egg sac silk of Araneus diadematus reached values of 30-40\%, much lower than the strains measured here (Van Nimmen, 2005b). Egg sac threads from Nephila clavipes extended to $24 \pm 2 \%$ their initial lengths and the maximum stress was observed to be $1.3 \pm 0.2 \mathrm{GPa}$, whereas for Araneus gemmoides, these values were respectively $19 \pm 2 \%$ and $2.3 \pm 0.2 \mathrm{GPa}$ (Stauffer, 1994).

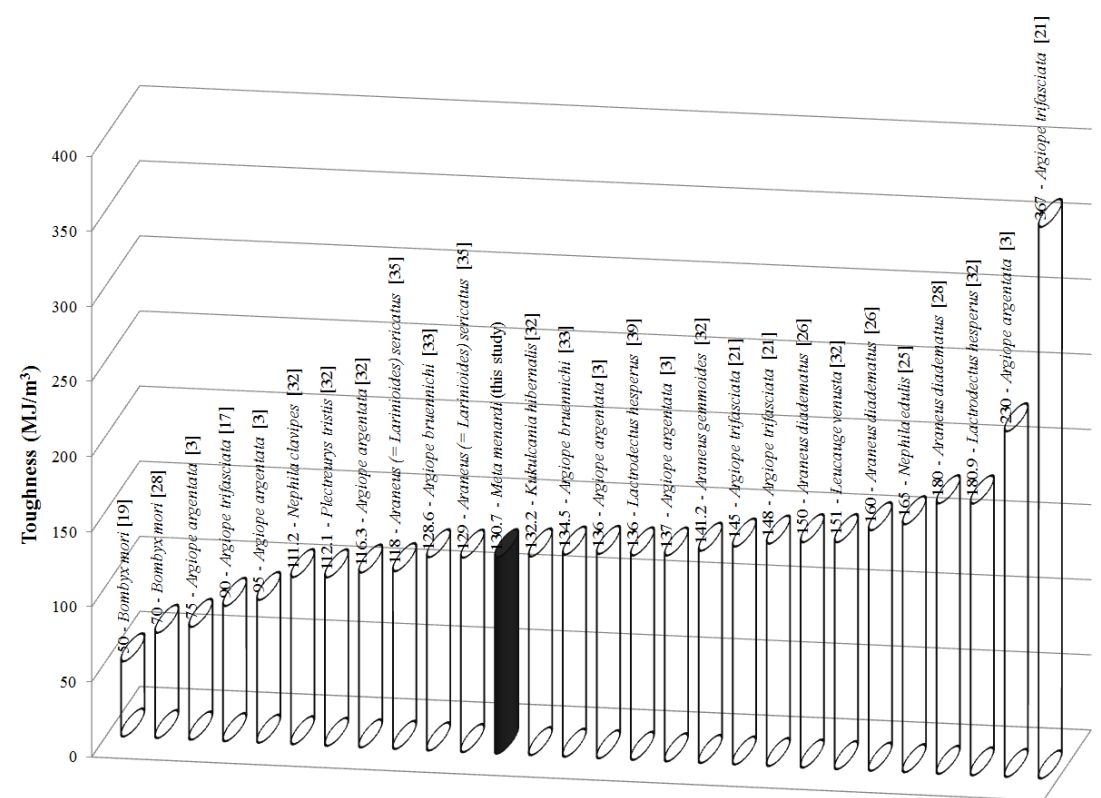

Figure 8.9 The maximum toughness of different types of (mainly spider) silks. 


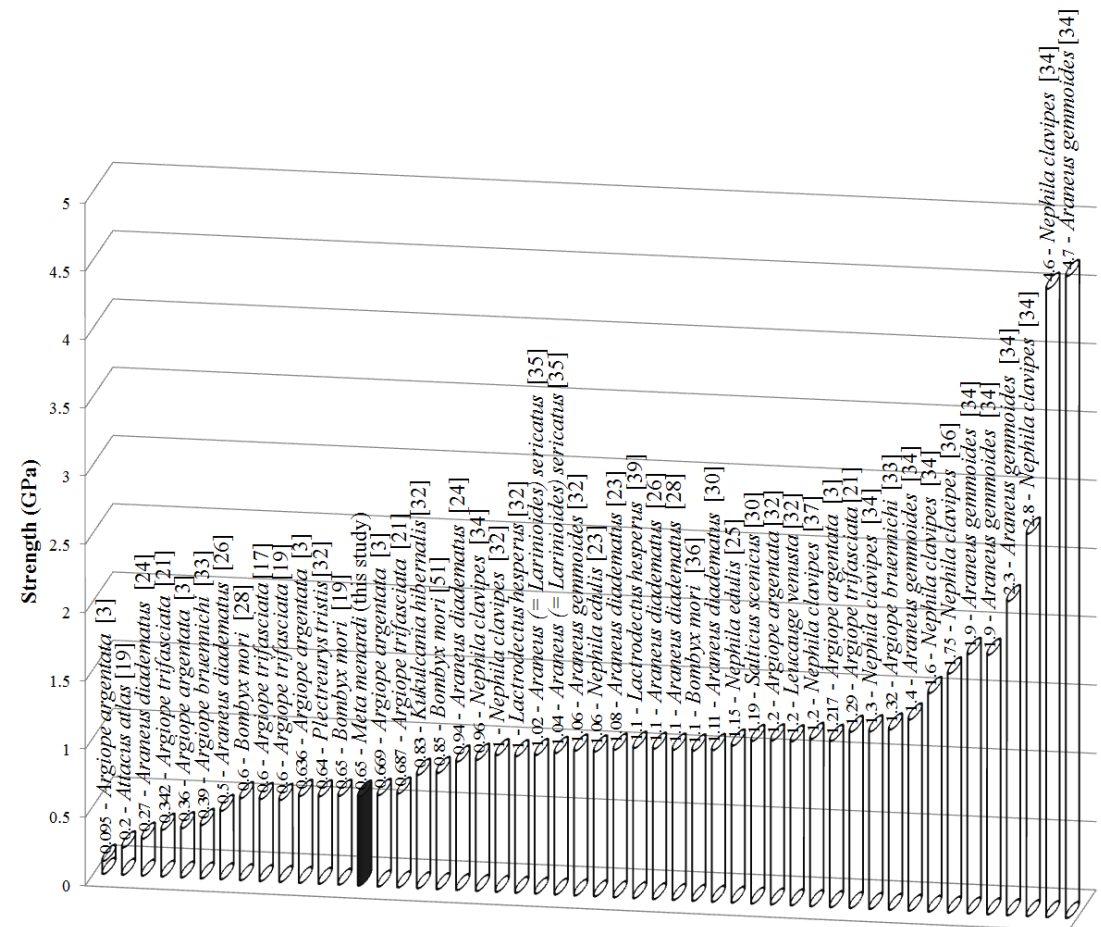

Figure 8.10 The maximum strength of different types of (mainly spider) silks.

The failure stresses of our stalks are much inferior to these, but the strains sustained by our stalks are much higher, which is most likely due to physical interactions within the stalks and the type of deformation occurring at the nanoscale. Bundles of dragline and minor ampullate silk composed of 100 threads were also tested (Stauffer, 1994) and show a wide range over which breaking occurred; these authors were not able to determine a useful value for the tensile strength of the fiber bundles due to the great variety in thread diameters in the bundle (Stauffer, 1994). We propose a solution to this problem using Weibull statistics, and our results ( $m$ is in the range from 1.5 to 1.8 , and $\sigma_{0}$ is in the range from 0.33 to $0.41 \mathrm{GPa}$ ) are consistent with the values of the shape $(m)$ and scale $\left(\sigma_{0}\right)$ parameters of the Weibull parameters of 3.4 and 0.6 GPa for the dragline of Argiope trifasciata (Perez-Rigueiro, 2001; Perez-Rigueiro, 1998) and 5.7 and 0.4 GPa for the silkworm cocoons of Bombyx mori (Perez-Rigueiro, 1998), respectively.

The large standard deviation in the values of our stress and strain results within the two groups of stalks might be due to differences in terms of diameter, 


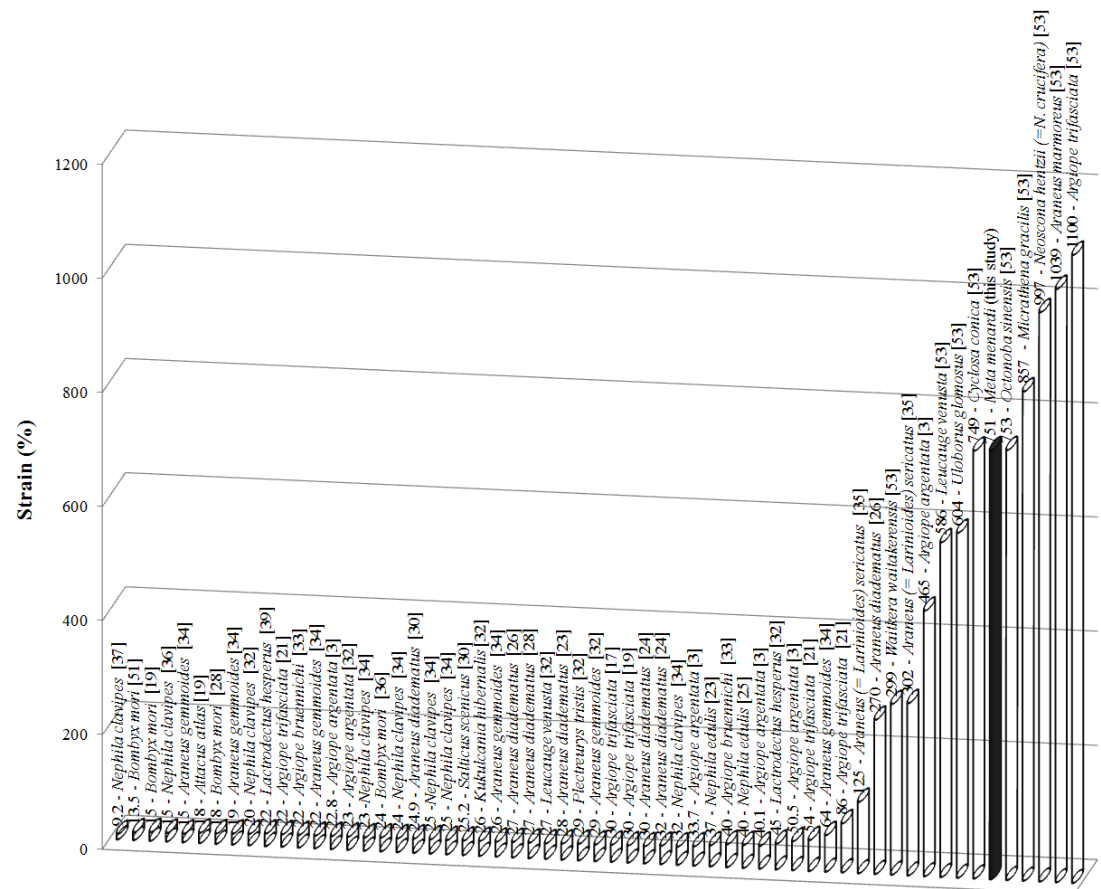

Figure 8.11 The maximum strain of different types of (mainly spider) silks, showing the record for egg sac silk stalks observed in the present study

number of threads, and the physical condition of the stalks, which may have affected performance (see Table 8.3). All stalks were obtained from the natural habitat of the spider where humidity and temperature play an important role. Moisture has been observed to induce thread supercontraction causing them to tighten up (Guinea, 2003). The temperature of the caves was roughly $9 \pm$ $2^{\circ} \mathrm{C}$, whereas the tests were performed in an environment at a much higher temperature, which might have caused the fibers to change from their natural state. The tests were also performed a couple of days after collection and were maintained in the laboratory at different conditions, which might have caused the modification or loss of some thread properties.

We observe that, the higher the stress sustained by the stalk, the lower the maximum strain before breakage. If strain reached high values the peak stresses did not exceed $0.64 \mathrm{GPa}$. In this case, we assume that the thread deformed in a rubber-like way, extending to significant lengths, due to physical interactions (Pugno, 2010; Allmeling, 2006; Dal Pra, 2005; Dal Pra, 2006) between the threads composing the stalk. 


\begin{tabular}{|c|c|c|c|c|c|c|c|c|c|c|}
\hline 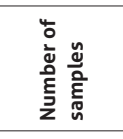 & 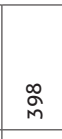 & $\stackrel{\substack{\infty \\
\sim}}{-1}$ & 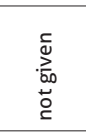 & 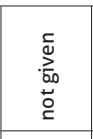 & i & $\stackrel{1}{\longrightarrow}$ & 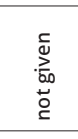 & 암 & $\stackrel{-1}{\circ}$ & 우 \\
\hline 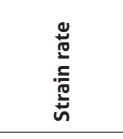 & 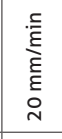 & 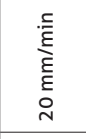 & 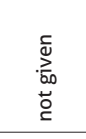 & 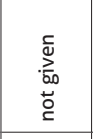 & 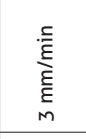 & 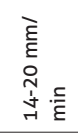 & 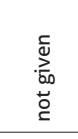 & 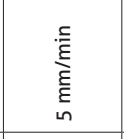 & 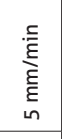 & 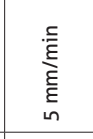 \\
\hline 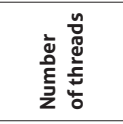 & $H$ & $H$ & 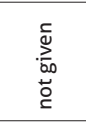 & 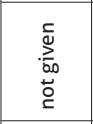 & $H$ & N & 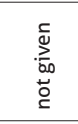 & $H$ & $H$ & $H$ \\
\hline$-^{\circ}$ & 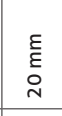 & 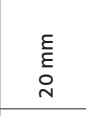 & 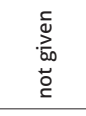 & 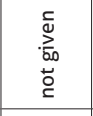 & 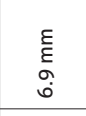 & 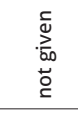 & 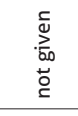 & 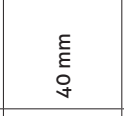 & $\begin{array}{l}E \\
\bar{E} \\
\sigma \\
\end{array}$ & 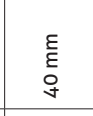 \\
\hline 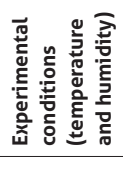 & 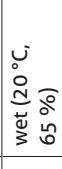 & 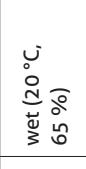 & 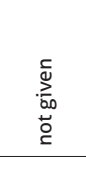 & 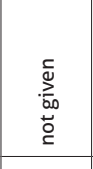 & 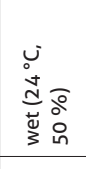 & 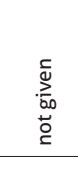 & 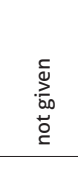 & 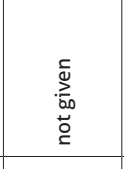 & 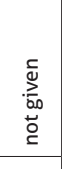 & 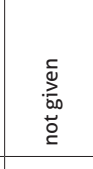 \\
\hline 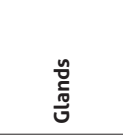 & 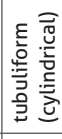 & 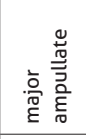 & 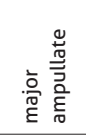 & 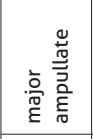 & 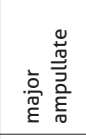 & 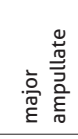 & 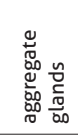 & 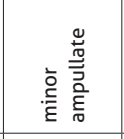 & 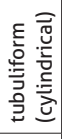 & 兽 \\
\hline 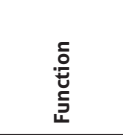 & 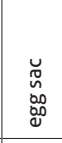 & 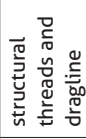 & 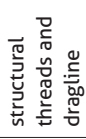 & 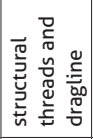 & 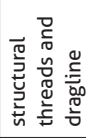 & 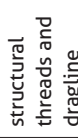 & 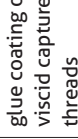 & 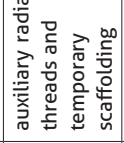 & 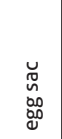 & 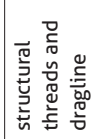 \\
\hline 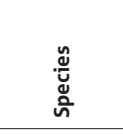 & 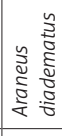 & 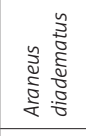 & 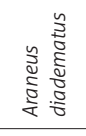 & 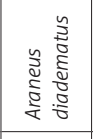 & 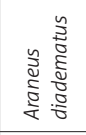 & 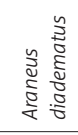 & 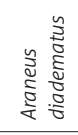 & 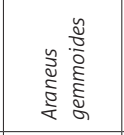 & 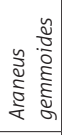 & 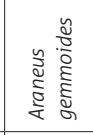 \\
\hline 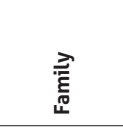 & 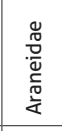 & 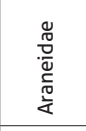 & 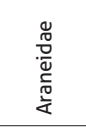 & 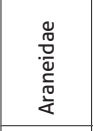 & $\begin{array}{l}\frac{\pi}{\pi} \\
\frac{\pi}{\pi} \\
\frac{\pi}{\pi} \\
\frac{\pi}{4} \\
\end{array}$ & 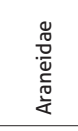 & 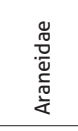 & 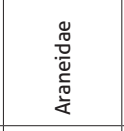 & $\begin{array}{l}\frac{0}{0} \\
\frac{5}{00} \\
\frac{50}{4} \\
\end{array}$ & 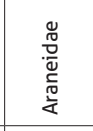 \\
\hline 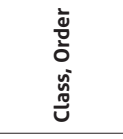 & 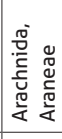 & 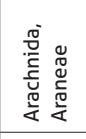 & 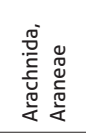 & 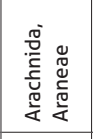 & 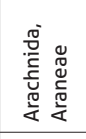 & 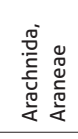 & 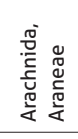 & 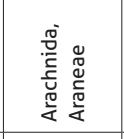 & 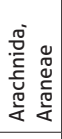 & 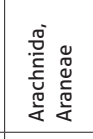 \\
\hline & 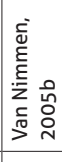 & 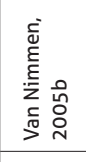 & 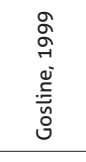 & 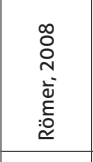 & 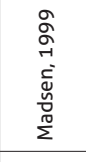 & 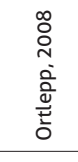 & 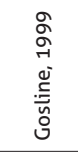 & 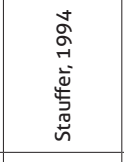 & 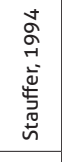 & 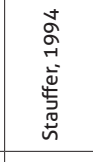 \\
\hline 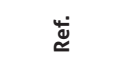 & $\stackrel{\Xi}{\Xi}$ & $\underset{\Xi}{\Xi}$ & ¿্ర & $\stackrel{\bar{\varpi}}{\widetilde{\Xi}}$ & $\overline{\mathbb{N}}$ & 品 & ఫ্ত & $\underset{\mathrm{m}}{\mathrm{F}}$ & 寻 & $\underset{\mathrm{f}}{\mathrm{J}}$ \\
\hline
\end{tabular}




\begin{tabular}{|c|c|c|c|c|c|c|c|c|c|}
\hline 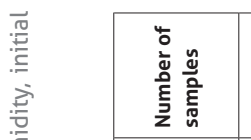 & $\stackrel{M}{N}$ & 우 & ㅇ & 8 & $\vec{y}$ & $m$ & M & $\tilde{\sigma}$ & $\stackrel{\infty}{N}$ \\
\hline 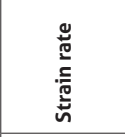 & 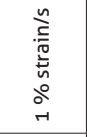 & 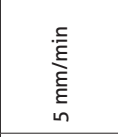 & 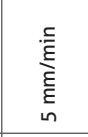 & 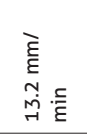 & 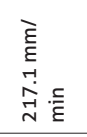 & 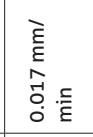 & 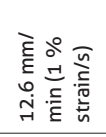 & 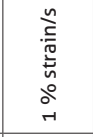 & 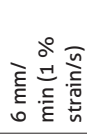 \\
\hline 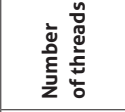 & 4 & $\underset{-}{\text { ¿ }}$ & $\stackrel{ }{\circ}$ & 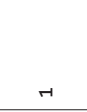 & H & 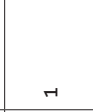 & H & H & 4 \\
\hline$-^{\circ}$ & 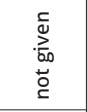 & $\begin{array}{l}\varepsilon \\
\varepsilon \\
\varepsilon \\
\dot{\sigma}\end{array}$ & $\begin{array}{l}\varepsilon \\
\varepsilon \\
\xi \\
\dot{y}\end{array}$ & $\begin{array}{l}\underset{E}{E} \\
\stackrel{\leftrightarrow}{N}\end{array}$ & 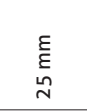 & 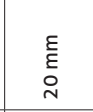 & $\begin{array}{l}E \\
\underset{\Sigma}{E} \\
\stackrel{N}{N}\end{array}$ & 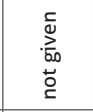 & 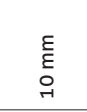 \\
\hline 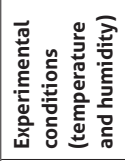 & 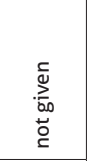 & 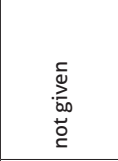 & 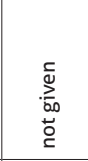 & 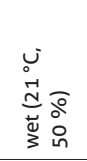 & 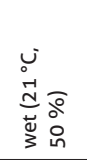 & 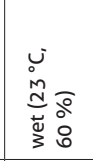 & 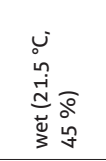 & 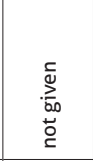 & 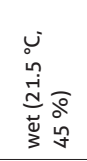 \\
\hline 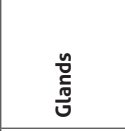 & 帝 & 总 & 总 & 惫 & 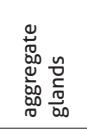 & 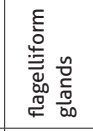 & 总 & 总 & 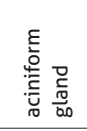 \\
\hline & 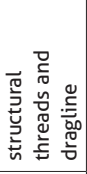 & 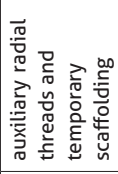 & 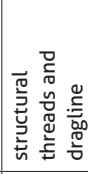 & 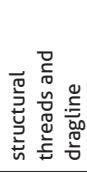 & 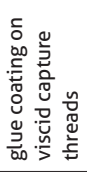 & 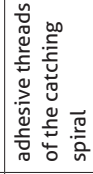 & 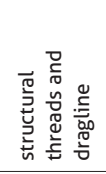 & 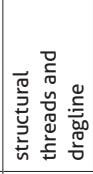 & 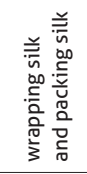 \\
\hline 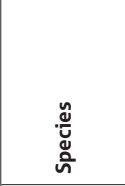 & 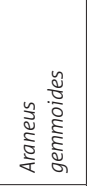 & 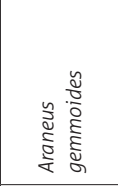 & 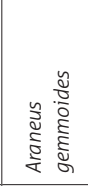 & 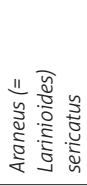 & 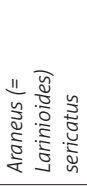 & 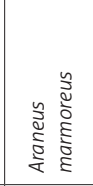 & 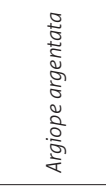 & 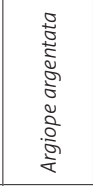 & 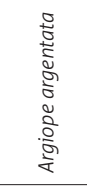 \\
\hline 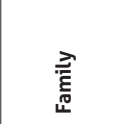 & 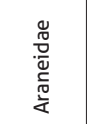 & $\begin{array}{l}\frac{0}{0} \\
\frac{\pi}{\pi} \\
\frac{\pi}{\pi} \\
\frac{\pi}{4}\end{array}$ & $\begin{array}{l}\frac{0}{\pi} \\
\frac{\pi}{\pi} \\
\frac{\pi}{\pi} \\
\frac{\pi}{4}\end{array}$ & 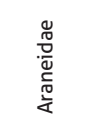 & 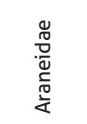 & 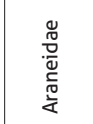 & $\begin{array}{l}\frac{\pi}{\pi} \\
\frac{\pi}{0} \\
\frac{\pi}{\pi} \\
\frac{\pi}{4} \\
\frac{\pi}{4}\end{array}$ & 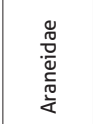 & 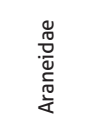 \\
\hline 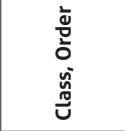 & 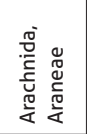 & 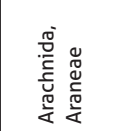 & 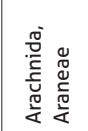 & 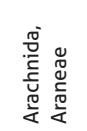 & 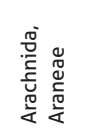 & 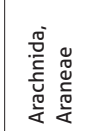 & 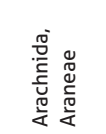 & 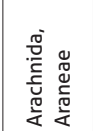 & 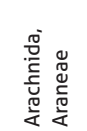 \\
\hline & 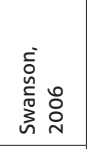 & 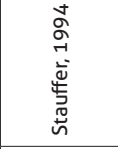 & 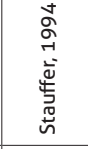 & 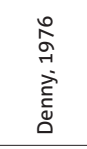 & 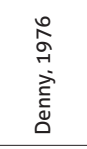 & 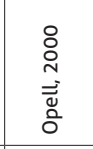 & 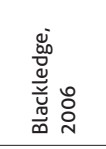 & 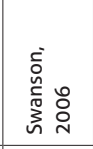 & 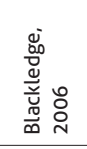 \\
\hline 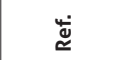 & $\widetilde{\widetilde{d}}$ & 趈 & 趈 & 忥 & 点 & 芯 & $\bar{m}$ & 胥 & $\overline{\underline{n}}$ \\
\hline
\end{tabular}




\begin{tabular}{|c|c|c|c|c|c|c|c|c|}
\hline 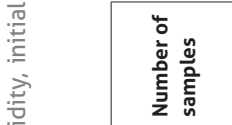 & $\widehat{\infty}$ & in & iे & t & t & $\stackrel{\infty}{\sim}$ & r & 엇 \\
\hline 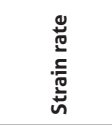 & 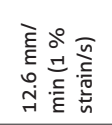 & 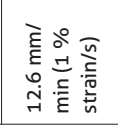 & 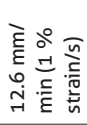 & $\begin{array}{l}\stackrel{c}{\xi} \\
\underline{\xi} \\
\stackrel{\xi}{\xi} \\
\circ\end{array}$ & 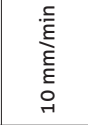 & 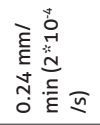 & 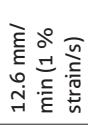 & 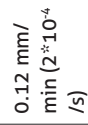 \\
\hline 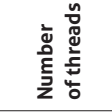 & $H$ & $H$ & H & $H$ & $H$ & $H$ & $H$ & $H$ \\
\hline - & $\begin{array}{l}\underset{E}{E} \\
\underset{N}{N}\end{array}$ & $\begin{array}{l}\underset{\xi}{E} \\
\underset{N}{N}\end{array}$ & $\begin{array}{l}\underset{N}{E} \\
\underset{N}{n}\end{array}$ & $\begin{array}{l}\underset{\varepsilon}{\varepsilon} \\
\stackrel{\sim}{\mathcal{N}}\end{array}$ & $\begin{array}{l}\varepsilon \\
\underset{\Sigma}{\varepsilon} \\
\stackrel{N}{N}\end{array}$ & $\begin{array}{l}\varepsilon \\
\stackrel{\varepsilon}{\varepsilon} \\
\stackrel{N}{N}\end{array}$ & $\begin{array}{l}\underset{E}{E} \\
\underset{N}{N}\end{array}$ & 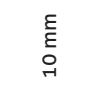 \\
\hline 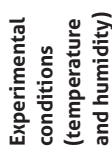 & 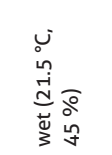 & 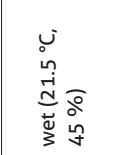 & 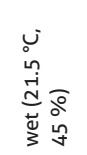 & 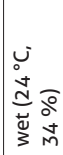 & 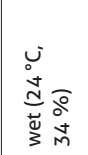 & 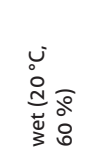 & 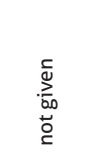 & 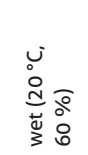 \\
\hline $\begin{array}{l}\stackrel{n}{0} \\
\frac{\pi}{0}\end{array}$ & 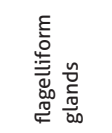 & 童 & 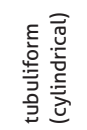 & 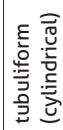 & 兽 & : & 兽 & 兽 \\
\hline 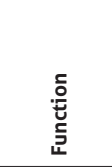 & 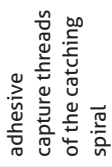 & 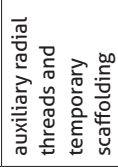 & 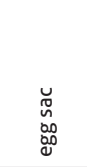 & 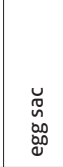 & 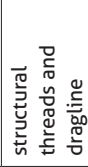 & 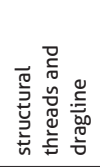 & 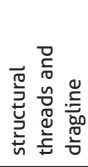 & 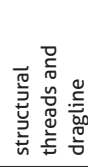 \\
\hline 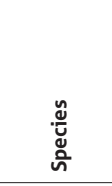 & 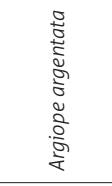 & 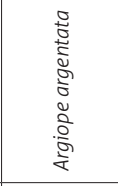 & 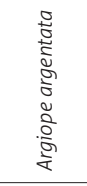 & 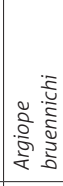 & 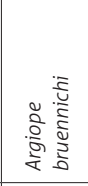 & 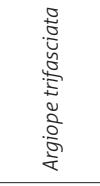 & 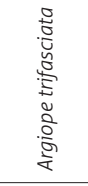 & 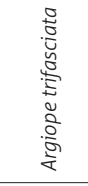 \\
\hline 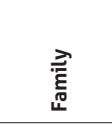 & 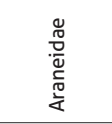 & 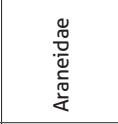 & 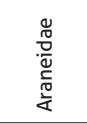 & 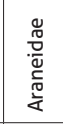 & 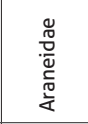 & 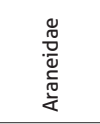 & 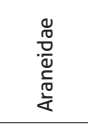 & $\begin{array}{l}\frac{0}{0} \\
\frac{\pi}{\pi} \\
\frac{\pi}{\pi} \\
\frac{\pi}{4}\end{array}$ \\
\hline 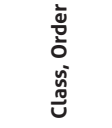 & 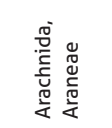 & 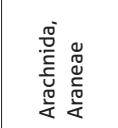 & 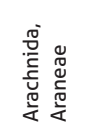 & 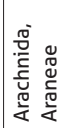 & 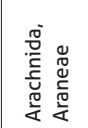 & 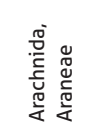 & 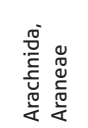 & 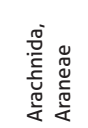 \\
\hline & 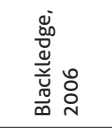 & 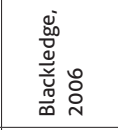 & 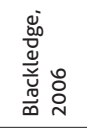 & $\begin{array}{l}\text { ¿ } \\
\text { N } \\
\vdots \\
\frac{0}{N} \\
\frac{\pi}{N}\end{array}$ & 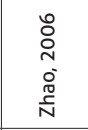 & 离 & 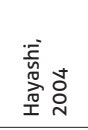 & $\begin{array}{l}\tilde{O} \\
\text { Oे } \\
\text { N } \\
0 \\
0\end{array}$ \\
\hline$\ddot{\check{\Xi}}$ & $\bar{m}$ & $\overline{\underline{n}}$ & $\overline{\underline{V}}$ & 志 & ్ㅗ & $\Xi$ & $\vec{I}$ & $\bar{\Xi}$ \\
\hline
\end{tabular}




\begin{tabular}{|c|c|c|c|c|c|c|c|c|}
\hline \multicolumn{9}{|c|}{$\mathrm{m}$} \\
\hline 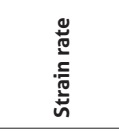 & 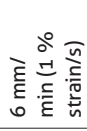 & 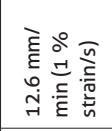 & 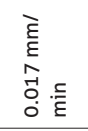 & 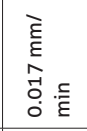 & 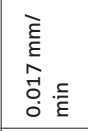 & 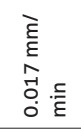 & 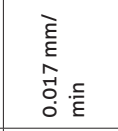 & 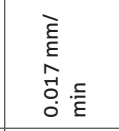 \\
\hline 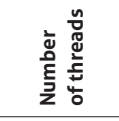 & $N$ & N & $H$ & $H$ & $H$ & H & $H$ & $H$ \\
\hline-0 & 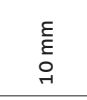 & $\begin{array}{l}\underset{E}{E} \\
\stackrel{N}{N}\end{array}$ & $\begin{array}{l}\varepsilon \\
\xi \\
\stackrel{N}{O} \\
\end{array}$ & $\begin{array}{l}\varepsilon \\
\varepsilon \\
\stackrel{\Sigma}{N}\end{array}$ & $\begin{array}{l}\varepsilon \\
\varepsilon \\
\stackrel{\Sigma}{N}\end{array}$ & $\begin{array}{l}\varepsilon \\
\xi \\
\stackrel{N}{O} \\
\end{array}$ & $\begin{array}{l}\varepsilon \\
\varepsilon \\
\stackrel{\Sigma}{N}\end{array}$ & 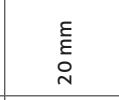 \\
\hline 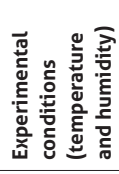 & 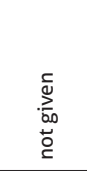 & 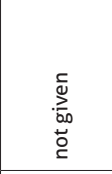 & 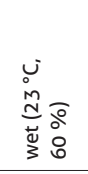 & 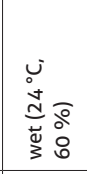 & 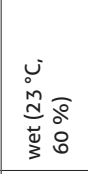 & 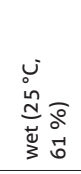 & 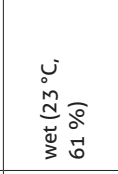 & $\begin{array}{l}3 \\
0 \\
0 \\
0 \\
0 \\
0 \\
0 \\
0\end{array}$ \\
\hline 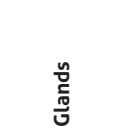 & 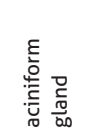 & 离 & 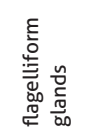 & 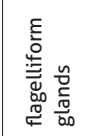 & 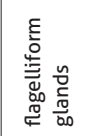 & 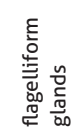 & 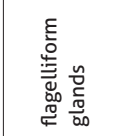 & 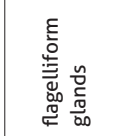 \\
\hline 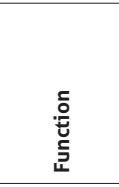 & 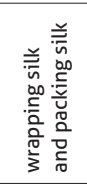 & 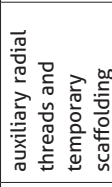 & 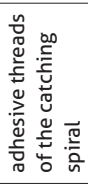 & 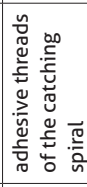 & 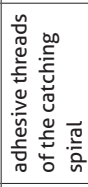 & 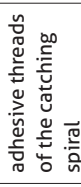 & 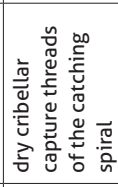 & 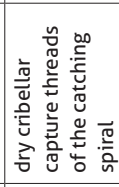 \\
\hline 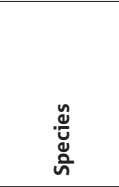 & 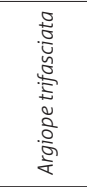 & 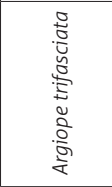 & 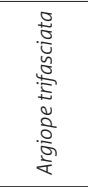 & 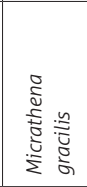 & 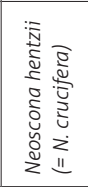 & 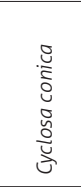 & 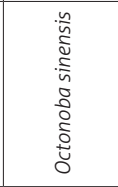 & 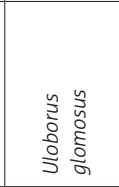 \\
\hline 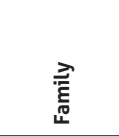 & $\begin{array}{l}\frac{0}{0} \\
\frac{0}{0} \\
\frac{0}{0} \\
\frac{\pi}{4} \\
\frac{\pi}{4}\end{array}$ & 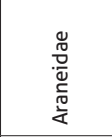 & 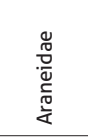 & 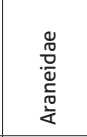 & $\begin{array}{l}\frac{0}{0} \\
\frac{0}{0} \\
\frac{0}{0} \\
\frac{0}{0} \\
\frac{\pi}{4}\end{array}$ & $\begin{array}{l}\frac{0}{0} \\
\frac{\pi}{0} \\
\frac{\pi}{0} \\
\frac{\pi}{4}\end{array}$ & 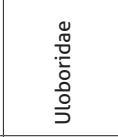 & $\begin{array}{l}0 \\
\frac{\pi}{0} \\
\frac{0}{0} \\
0 \\
0 \\
5\end{array}$ \\
\hline 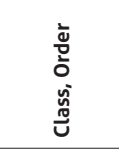 & 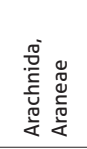 & 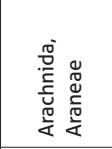 & 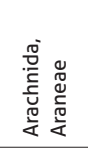 & 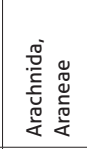 & 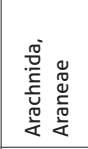 & 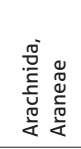 & 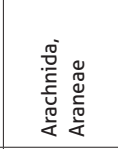 & 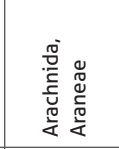 \\
\hline & $\begin{array}{l}\text { 蒿 } \\
\text { 离 } \\
\text { 耎 }\end{array}$ & 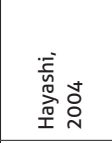 & $\begin{array}{l}\stackrel{\circ}{0} \\
\text { } \\
=\overline{0} \\
\text { o }\end{array}$ & $\begin{array}{l}\stackrel{0}{0} \\
\stackrel{\overline{0}}{0} \\
\text { ô }\end{array}$ & $\begin{array}{l}\stackrel{\circ}{0} \\
\stackrel{1}{=} \\
\stackrel{0}{0}\end{array}$ & 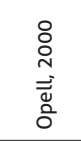 & 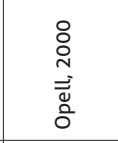 & 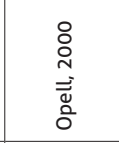 \\
\hline 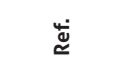 & $\vec{\Xi}$ & $\vec{\Xi}$ & હ & હ & હ్ & 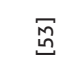 & 苞 & 芯 \\
\hline
\end{tabular}




\begin{tabular}{|c|c|c|c|c|c|c|c|c|c|}
\hline 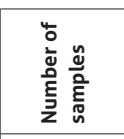 & $\mathrm{m}$ & $\stackrel{\sim}{\tilde{T}}$ & $\therefore$ & $\stackrel{\circ}{m}$ & $\overrightarrow{0}$ & $m$ & 욱 & $\stackrel{\circ}{\circ}$ & 욱 \\
\hline 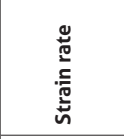 & 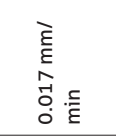 & 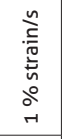 & 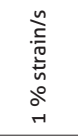 & 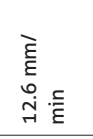 & 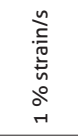 & 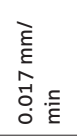 & 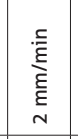 & 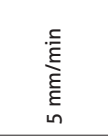 & 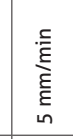 \\
\hline 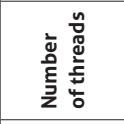 & H & $H$ & H & i & H & $H$ & 品 & $H$ & 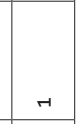 \\
\hline- & $\begin{array}{l}\text { E } \\
\stackrel{E}{E} \\
\stackrel{N}{N}\end{array}$ & 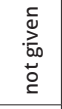 & 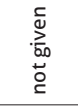 & $\underset{\sim}{\stackrel{E}{E}}$ & 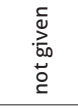 & 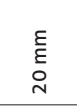 & 完 & 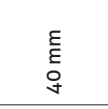 & 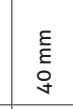 \\
\hline 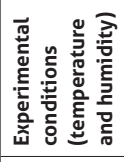 & 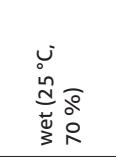 & 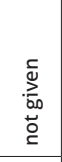 & 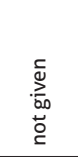 & 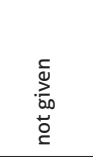 & 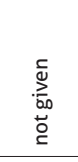 & 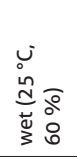 & 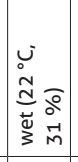 & 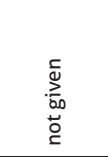 & 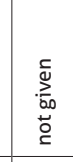 \\
\hline 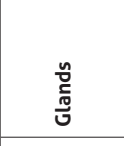 & 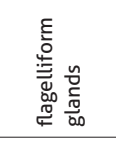 & 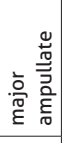 & 总 & 总 & 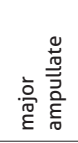 & 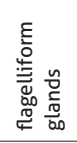 & 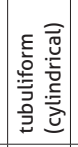 & 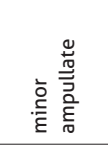 & 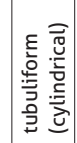 \\
\hline 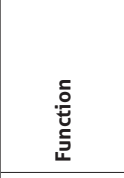 & 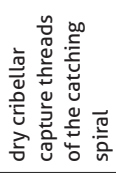 & 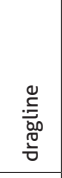 & 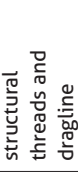 & 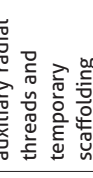 & 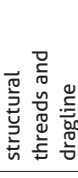 & 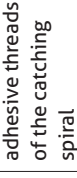 & 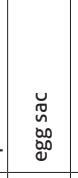 & 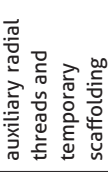 & 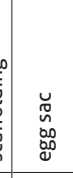 \\
\hline 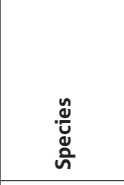 & 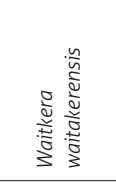 & 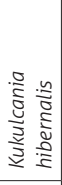 & 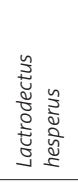 & 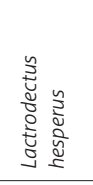 & 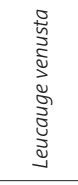 & 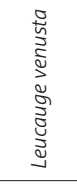 & 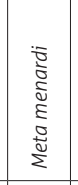 & 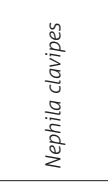 & 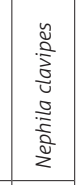 \\
\hline 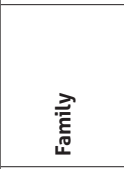 & 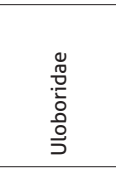 & 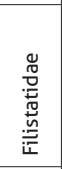 & 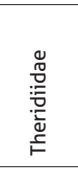 & 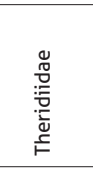 & 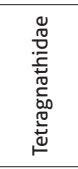 & 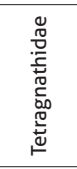 & 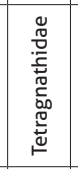 & 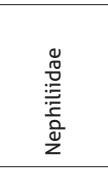 & 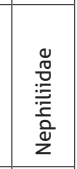 \\
\hline 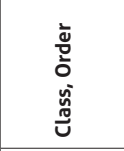 & 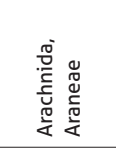 & 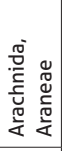 & 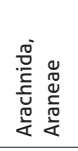 & 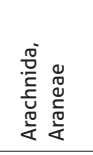 & 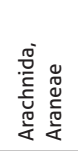 & 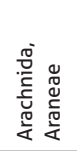 & 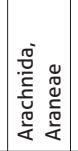 & 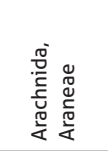 & 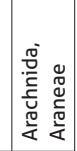 \\
\hline & 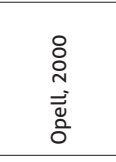 & 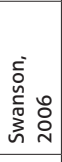 & 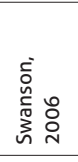 & 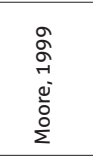 & 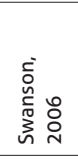 & $\begin{array}{l}\stackrel{\circ}{0} \\
\stackrel{0}{\vec{J}} \\
\stackrel{\Delta}{0}\end{array}$ & & 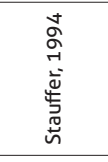 & 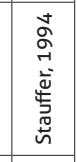 \\
\hline 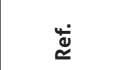 & $\stackrel{\bar{n}}{\underline{n}}$ & 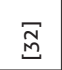 & $\overline{\tilde{c}}$ & 鸹 & $\overline{\tilde{\omega}}$ & 慶 & 号急 & $\underset{\mathrm{m}}{\stackrel{F}{0}}$ & 蛋 \\
\hline
\end{tabular}




\begin{tabular}{|c|c|c|c|c|c|c|c|c|c|}
\hline \multicolumn{10}{|l|}{ 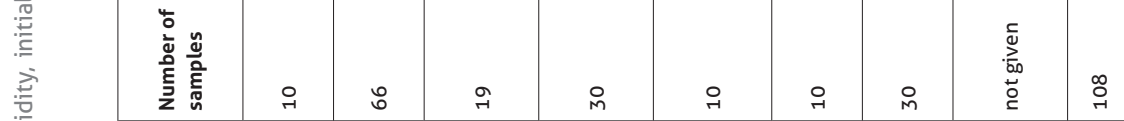 } \\
\hline 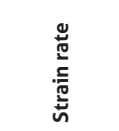 & 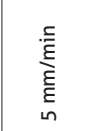 & 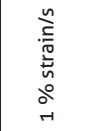 & 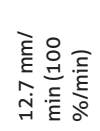 & 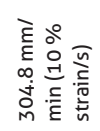 & 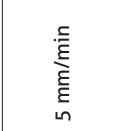 & 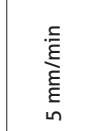 & $\begin{array}{l}\stackrel{c}{\xi} \\
\frac{\xi}{\xi} \\
\frac{\xi}{m}\end{array}$ & 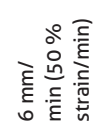 & 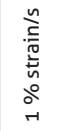 \\
\hline 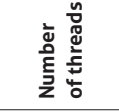 & $H$ & $H$ & $r$ & $r$ & ঃ & $\stackrel{\circ}{\circ}$ & H & H & $H$ \\
\hline - & $\begin{array}{l}\varepsilon \\
\varepsilon \\
\stackrel{\varepsilon}{\sigma}\end{array}$ & 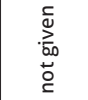 & $\begin{array}{l}\varepsilon \\
\stackrel{\varepsilon}{\xi} \\
\stackrel{\sim}{\mathcal{I}}\end{array}$ & $\begin{array}{l}\varepsilon \\
\xi \\
\infty \\
\infty \\
i\end{array}$ & $\begin{array}{l}\varepsilon \\
\xi \\
\stackrel{\xi}{q}\end{array}$ & $\begin{array}{l}\varepsilon \\
\varepsilon \\
\xi \\
\stackrel{\sigma}{\sigma}\end{array}$ & $\begin{array}{l}\varepsilon \\
\xi \\
\xi \\
\sigma\end{array}$ & $\begin{array}{l}\underset{\mathcal{E}}{E} \\
\underset{\sim}{\mathrm{V}}\end{array}$ & 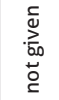 \\
\hline 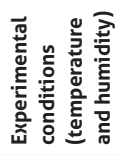 & 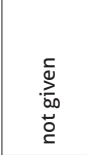 & 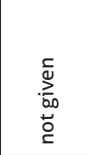 & 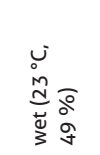 & 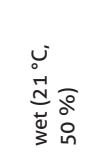 & 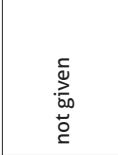 & 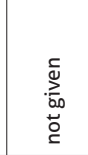 & 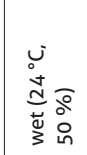 & 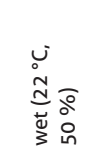 & 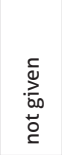 \\
\hline 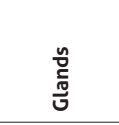 & 总 & 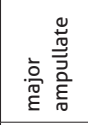 & 兽 & 总 & 总 & 흘 & 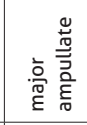 & 兽 & 兽 \\
\hline 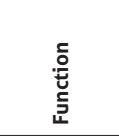 & 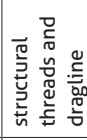 & 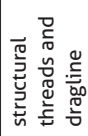 & 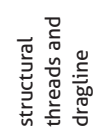 & 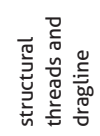 & 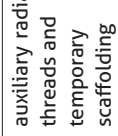 & 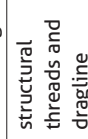 & 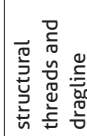 & 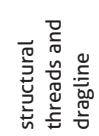 & 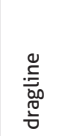 \\
\hline 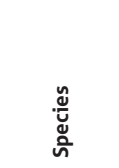 & 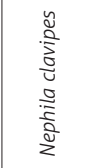 & 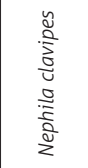 & 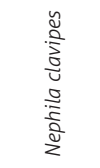 & 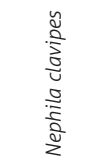 & 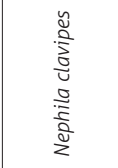 & 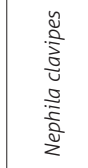 & $\begin{array}{l}\frac{n}{5} \\
\frac{a}{2} \\
\frac{9}{\tilde{z}} \\
\frac{\bar{a}}{2}\end{array}$ & 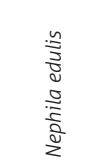 & 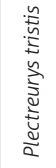 \\
\hline 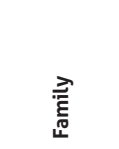 & 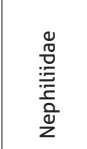 & 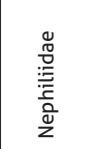 & 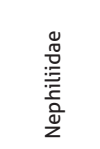 & 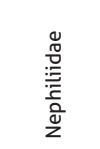 & $\begin{array}{l}\frac{0}{0} \\
: \frac{0}{0} \\
: \frac{0}{0} \\
\frac{0}{2}\end{array}$ & 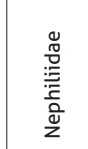 & 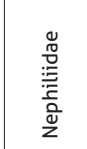 & 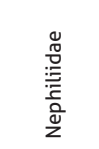 & 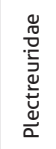 \\
\hline 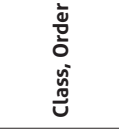 & 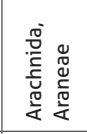 & 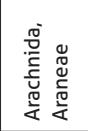 & 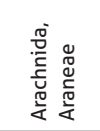 & 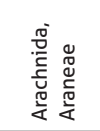 & 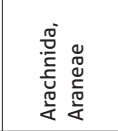 & 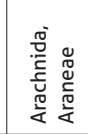 & 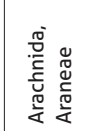 & 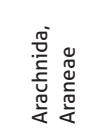 & 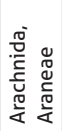 \\
\hline & 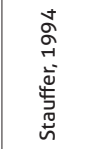 & 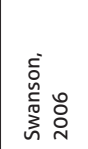 & $\begin{array}{l}\text { 离 } \\
\text { 总 } \\
\text { 号 } \\
\text { 品 }\end{array}$ & 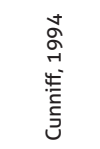 & 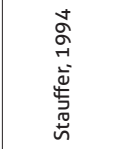 & 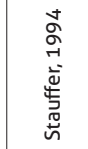 & 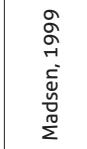 & 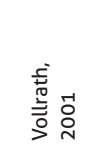 & 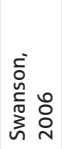 \\
\hline 巡 & 趈 & స్త్ర & 容 & 巟 & 疋 & 予 & $\overline{\mathbb{Z}}$ & $\sqrt[\underline{N}]{\bar{N}}$ & స్ల \\
\hline
\end{tabular}




\begin{tabular}{|c|c|c|c|c|c|c|}
\hline 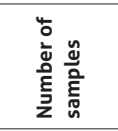 & in & 우 & $\begin{array}{l}\sum_{\bar{\nu}} \\
\text { के } \\
\stackrel{0}{c}\end{array}$ & 엄 & 아 & $\stackrel{\circ}{N}$ \\
\hline 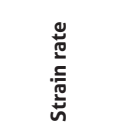 & 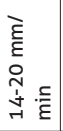 & 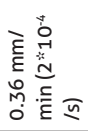 & 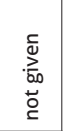 & 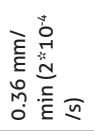 & 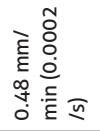 & 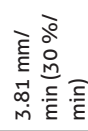 \\
\hline 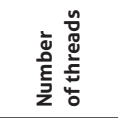 & N & 4 & 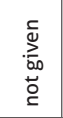 & 4 & $H$ & $H$ \\
\hline$-^{0}$ & 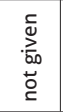 & 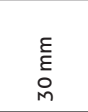 & 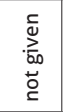 & 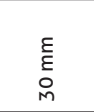 & 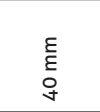 & 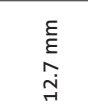 \\
\hline 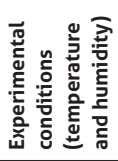 & 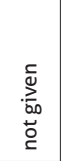 & 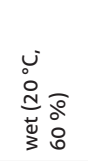 & 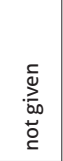 & 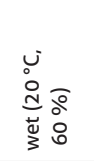 & 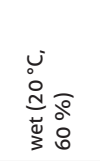 & 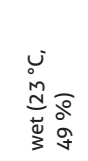 \\
\hline $\begin{array}{l}\frac{n}{0} \\
\frac{n}{5}\end{array}$ & 总 & 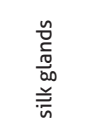 & $\begin{array}{l}\frac{n}{0} \\
\frac{0}{00} \\
\frac{5}{\bar{n}}\end{array}$ & $\begin{array}{l}\frac{n}{0} \\
\frac{0}{00} \\
\frac{5}{\bar{n}}\end{array}$ & 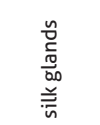 & 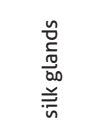 \\
\hline 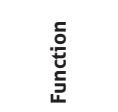 & 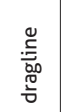 & 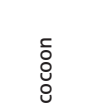 & 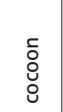 & 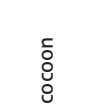 & 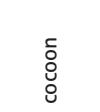 & 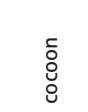 \\
\hline 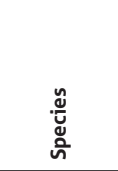 & 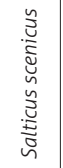 & 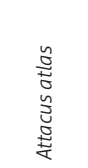 & 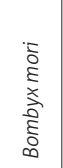 & 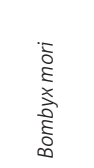 & 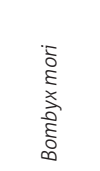 & 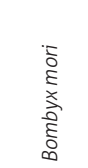 \\
\hline $\begin{array}{l}\vec{z} \\
\stackrel{\vec{E}}{\bar{E}} \\
\stackrel{5}{5}\end{array}$ & 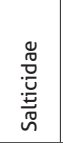 & 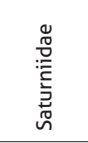 & 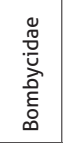 & 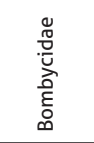 & 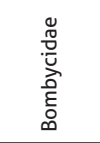 & 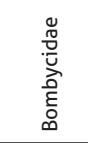 \\
\hline 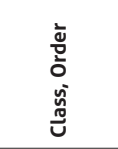 & 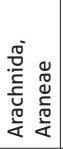 & 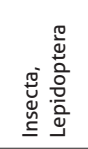 & 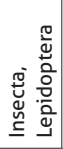 & 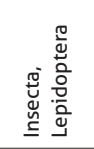 & 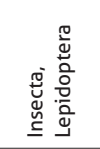 & 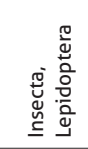 \\
\hline & 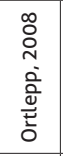 & 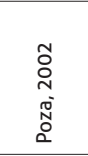 & 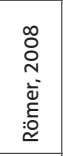 & 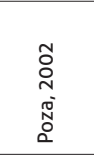 & 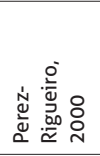 & 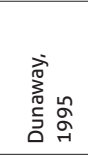 \\
\hline 巡 & 穴 & $\bar{\Xi}$ & $\stackrel{\infty}{\underset{V}{\infty}}$ & $\bar{\Xi}$ & 莺 & 灾 \\
\hline
\end{tabular}




\subsection{Conclusion}

The tensile properties and the Weibull shape and scale parameters of egg sac silk stalks of Meta menardi, obtained directly from their natural habitat, were determined in the present study. The results differ significantly compared with other tensile tests on spider silk. When compared with egg sac silk from other species of orbweb weavers, dragline silk, or minor ampullate silk, the presented results for maximum strain are much higher, up to $750 \%$ than those previously reported, suggesting the discovery of the most stretchable egg sac silk stalk ever tested. Such significant elongations suggests a huge unrolling microscopic mechanism of the macroscopic stalk that, as a continuation of the protective egg sac, is expected to be composed by of very densely and randomly packed fibers. 
An Experimental Study on Adhesive or Anti-adhesive, Bio-inspired Experimental Nanomaterials 